\title{
QUERMASS-INTERACTION PROCESSES: CONDITIONS FOR STABILITY
}

\author{
W. S. KENDALL, ${ }^{*}$ University of Warwick \\ M. N. M. VAN LIESHOUT, ${ }^{* *} C W I$ \\ A. J. BADDELEY, ${ }^{* * *}$ University of Western Australia
}

\begin{abstract}
We consider a class of random point and germ-grain processes, obtained using a rather natural weighting procedure. Given a Poisson point process, on each point one places a grain, a (possibly random) compact convex set. Let $\Xi$ be the union of all grains. One can now construct new processes whose density is derived from an exponential of a linear combination of quermass functionals of $\Xi$. If only the area functional is used, then the area-interaction point process is recovered. New point processes arise if we include the perimeter length functional, or the Euler functional (number of components minus number of holes). The main question addressed by the paper is that of when the resulting point process is well-defined: geometric arguments are used to establish conditions for the point process to be stable in the sense of Ruelle.

Keywords: Area-interaction point process; Boolean model; germ-grain model; Markov point process; Minkowski functional; quermass integral; semi-Markov random closed set; spatial point process
\end{abstract}

AMS 1991 Subject Classification: Primary 62M30; 60G55; 60K35

The analysis of digital images and spatial patterns calls for tractable stochastic models of random sets and point processes. In this paper, we investigate new point process and germ-grain models which are constructed by weighting a Poisson point process (or germgrain process) using exponentials of (sums of) quermass integrals (Minkowski functionals) of a Boolean model based on the reference random process. These functionals are obtained from local geometric measurements including set volume and integrals of curvature over the boundary, and include the Euler-Poincaré characteristic.

In the point process case the model under investigation generalises the Widom-Rowlinson penetrable spheres model [65] the area-interaction point process [4] and the morphological model in [34, 37, 38].

In this paper our main focus will be on the conditions under which planar quermassinteraction processes are stable in the sense of Ruelle (inequality (9) in Section 2.1 below). This is important because stability is an accessible condition for the density to be proper (to

\footnotetext{
Received 11 September 1996; revision received 25 February 1998.

* Postal address: Department of Statistics, University of Warwick, Coventry, CV4 7AL, UK.

Email address: w.s.kendall@warwick.ac.uk

** Postal address: Centre for Mathematics and Computer Science, PO Box 94079, 1090 GB, Amsterdam, The Netherlands.

*** Postal address: Department of Mathematics, University of Western Australia, Nedlands, WA 6907, Australia.
} 
integrate to unit total mass rather than infinity), as well as being useful when studying the behaviour of the process (for example, whether its definition can be extended from bounded windows to the whole plane) and when devising simulation algorithms. Stability has already been established for the special case of area-interaction [4]; we shall establish it in greater generality, with particular attention to the Euler-Poincaré characteristic. Our arguments are basically geometric covering arguments of a rather non-standard form, essentially elementary but of some intrinsic geometric interest. In further papers we hope to develop inferential and simulation theory as well as to explore the utility of this class of models in applications.

The paper is divided into seven sections: Section 1 covers preliminaries on stochastic geometry; Section 2 defines quermass-interaction germ-grain models and random sets; Section 3 begins the discussion of the important planar case, which introduces the main question to be dealt with in this initial study, namely the range of permissible parameter values under which the Euler-Poincaré characteristic yields a stable germ-grain process; Section 4 shows stability when grains are planar disks; Section 5 considers the case when grains are convex polygons, in which case a lower bound on interior angles and side-lengths is needed; finally Section 6 indicates our plans for future investigation of these point processes, including simulation and inference issues.

\section{Preliminaries}

In this section we briefly summarize relevant facts from the theories of Markov point processes, Boolean models, and quermass integrals.

\subsection{Point processes}

The basic reference process is a (stationary) Poisson point process in a bounded observation region $\&$. This can be understood to exhibit spatial independence in the sense that points do not interact with each other. More specifically, given that there are $n$ points, these are independent and uniformly distributed over $\&$. The total number of points in $\&$ is Poisson distributed with mean proportional to the area of $\delta$. The constant of proportionality is called the intensity. The area measure can be replaced by any finite diffuse measure $\mu$, yielding an inhomogeneous Poisson point process with intensity measure $\mu$.

One can define other processes by specifying their densities with respect to the Poisson process. For a process defined in this way, with density $p(\cdot)$, the distribution $\left(q_{0}, q_{1}, q_{2}, \ldots\right)$ of the total number of points is given by

$$
q_{n}=\frac{\mathrm{e}^{-\mu(\S)}}{n !} \int_{\S} \ldots \int_{\S} p\left(\left\{x_{1}, \ldots, x_{n}\right\}\right) \mathrm{d} \mu\left(x_{1}\right) \ldots \mathrm{d} \mu\left(x_{n}\right)
$$

and, given $N=n$, the joint conditional probability density of the point pattern is

$$
p_{n}\left(x_{1}, \ldots, x_{n}\right)=\mathrm{e}^{-\mu(\delta)} p\left(\left\{x_{1}, \ldots, x_{n}\right\}\right) /\left(n ! q_{n}\right)
$$

where the reference measure is provided by the product measure $\mu^{n}$ on $8^{n}$.

It will be convenient to impose conditions on the density.

Definition 1.1. Let $\sim$ be a symmetric relation on $\&$. Then a density $p(\cdot)$ defines a Markov point process [53] if for all patterns $\boldsymbol{x}=\left\{x_{1}, \ldots, x_{n}\right\}$ such that $p(\boldsymbol{x})>0$

$$
p(\boldsymbol{y})>0 \text { for all } \boldsymbol{y} \subset \boldsymbol{x}
$$


(M2) the Papangelou conditional intensity

$$
\lambda(u ; \boldsymbol{x})=\frac{p(\boldsymbol{x} \cup\{u\})}{p(\boldsymbol{x})}
$$

depends only on $u$ and $\left\{x_{i}: u \sim x_{i}\right\}$.

Note that any strictly positive density $p(\boldsymbol{x})$ can be reconstructed from the Papangelou conditional intensity up to a constant factor (and this means that $p(\cdot)$ is completely defined once the conditional intensity $\lambda(\cdot ; \cdot)$ is prescribed, since the density $p(\cdot)$ must have unit total mass).

A generalization of Definition 1.1 can be obtained by allowing the relation to depend on the configuration [5].

The celebrated Hammersley-Clifford theorem [5, 6, 8, 16, 51, 53, 59] gives a simple interpretation in terms of interpoint interactions. A process with density $p(\cdot)$ is a Markov point process if and only if

$$
p(\boldsymbol{x})=\prod_{\text {cliques } \boldsymbol{y} \subseteq \boldsymbol{x}} q(\boldsymbol{y})=\alpha \prod_{\substack{\text { cliques } \boldsymbol{y} \subseteq \boldsymbol{y} \\ \boldsymbol{y} \neq \varnothing}} q(\boldsymbol{y})
$$

for arbitrary non-negative interaction functions $q(\cdot)$, save that $\alpha=q(\varnothing)$ is determined by the requirement that the total integral of $p(\cdot)$ equals 1 .

Because of property (M2), Markov point processes are natural models for problems involving derivation of conditional probabilities and also are easy to simulate using Markov chain Monte Carlo methods, and hence are amenable to iterative statistical techniques [7, 9, $15,33,40]$.

\subsection{Boolean models}

The Boolean model and its associated Poisson germ-grain model are defined in [60]. Briefly, a set called a grain is placed at each point of a (possibly inhomogeneous) Poisson point process of germs in Euclidean space. Different random grains are random compact sets which are independent of each other conditional on the realization of the point process of germs. We consider two cases:

(a) Different grains are independent both of each other and of locations, and follow the same distribution $v$, and (more generally)

(b) The distribution of a grain depends continuously with respect to the Hausdorff metric on the location of the respective germ, but each grain is independent of other locations and other grains.

This produces a Poisson marked point process, by marking the germ process with the grains. Finally, the Boolean model is the random set obtained by the union of all the grains. In this paper, unless specifically stated otherwise, we will assume that the grains are ovoids (that is to say, non-empty convex compact sets with non-empty interior).

By virtue of the Choquet theorem [36, Theorem 2-2-1], a random closed set $\Xi$ is determined by its avoidance function on $\mathcal{K}$, the family of compact sets, defined by

$$
Q(K)=\mathbb{P}(\Xi \cap K=\varnothing) .
$$


For a Boolean model based on a homogeneous Poisson point process with random grains (case $(a))$

$$
Q(K)=\exp [-\beta \mathbb{E} \text { volume }(K \oplus \check{Z})]
$$

where $\mathbb{E}$ denotes the expectation with respect to $\nu$ of the typical grain $Z, \beta$ is the intensity of the underlying Poisson point process and $\oplus$ is the Minkowski sum.

We should distinguish between the case where we can observe both the germs and the grains (for example in the area-interaction point process model described below where the configuration of grains can be deduced from the configuration of germ points) and the random set case where only the union of the grains is observed and not the underlying germ process. This distinction has important consequences for statistical inference, which has to be based only on observable quantities. However it does not affect the arguments of this paper, which focus on stability and existence considerations.

\subsection{Quermass integrals}

The quermass integrals or Minkowski functionals are fundamental concepts of geometry [18, 58] generalising the notions of area and perimeter. In $d$ dimensions and for $j \leq d-1$, they are defined for ovoids $K \in C(\mathcal{K})$ by

$$
W_{j}^{d}(K)=\frac{b_{d}}{b_{d-j}} \int_{L_{j}} v_{d-j}\left(\operatorname{proj}_{S^{\perp}}(K)\right) \mathrm{d} \mu_{j}(S)
$$

where $L_{j}$ is the class of all $j$-dimensional subspaces $S, \mu_{j}$ is the unique probability measure on $L_{j}$ that is invariant under rigid motions, $\operatorname{proj}_{S^{\perp}}$ is the map projecting onto $S^{\perp}$ the subspace orthogonal to $S$, and $v_{j}$ is Lebesgue measure on $j$-dimensional space. Furthermore, $b_{d}=$ $\pi^{d / 2} / \Gamma(1+d / 2)$ is the $d$-volume of the $d$-dimensional unit ball. Equivalently, $W_{j}^{d}(K)$ for $j \leq d-1$ is the invariant measure of the set of all affine $j$-dimensional subspaces intersecting $K$, normalised so that the unit ball $B=B(0,1)$ has $W_{j}^{d}(B)=b_{d}$. Finally, set $W_{d}^{d}(\cdot) \equiv b_{d}$.

A different but equivalent definition is via the Steiner formula

$$
v_{d}(K \oplus B(0, t))=\sum_{j=0}^{d}\left(\begin{array}{l}
d \\
j
\end{array}\right) W_{j}^{d}(K) t^{j} .
$$

Interesting special cases include the following:

$W_{0}^{d}(K)$ is the Lebesgue measure $v_{d}(K)$ of $K$;

$d W_{1}^{d}(K)$ is the surface area of $K$;

$d W_{2}^{d}(K)$ is the integral mean curvature over the boundary of $K$;

and $W_{d-1}^{d}(K)=\left(b_{d} / 2\right) \bar{b}(K)$ is proportional to the mean breadth $\bar{b}(K)$ of the ovoid $K$.

If the boundary $\partial K$ is sufficiently regular, for example if it is possible to define at each point $t \in \partial K$ the $d-1$ principal curvatures, then the Minkowski functionals admit simple integral representations using symmetric functions of these curvatures (see Matheron [36]). Thus for example

$$
d W_{2}^{d}(K)=\int_{\partial K} m(t) \mathrm{d} t
$$

where $m(t)$ is the mean curvature at $t$. 
Let $\psi$ be a functional defined for all ovoids. It is called $C$-additive if

$$
\psi\left(K_{1} \cup K_{2}\right)=\psi\left(K_{1}\right)+\psi\left(K_{2}\right)-\psi\left(K_{1} \cap K_{2}\right)
$$

for any ovoids $K_{1}, K_{2} \in C(\mathcal{K})$ for which the union is again an ovoid ( $K_{1} \cup K_{2} \in C(\mathcal{K})$ ). The Minkowski functionals are $\mathrm{C}$-additive, and also increasing, continuous with respect to Hausdorff distance and invariant under rigid motions. Hadwiger's characterization theorem delivers a converse to this observation: any sufficiently well-behaved ovoid-functional can be written as a linear combination of Minkowski functionals. More specifically

Theorem 1.2. (Hadwiger's characterization theorem [18]) Suppose that $\psi$ is a $C$-additive ovoid functional (hence $\psi(K)$ is defined for $K \in C(\mathcal{K})$ ) which is continuous with respect to the Hausdorff metric on $C(\mathcal{K})$ and is invariant under rigid motions. Then it can be written as a linear combination of quermass integrals

$$
\psi=\sum_{j=0}^{d} a_{j} W_{j}^{d}
$$

where the coefficients $a_{j}$ are uniquely defined. (If 'continuous' is replaced by 'increasing' (with respect to set-inclusion) or 'non-negative' then the same statement holds under the further condition that the $a_{j}$ are non-negative.)

We intend to use quermass integrals to define new germ-grain models. Hence we will be interested in evaluation of quermass integrals on finite unions of convex compact sets, which form the convex ring $\mathcal{R}$. The quermass integrals can be extended onto the convex ring in several ways. The most direct is the additive extension

$$
W_{j}^{d}(K)=\frac{b_{d}}{b_{d-j}} \int_{L_{j}}\left(\int_{S^{\perp}} \chi\left(K \cap S_{x}\right) \mathrm{d} x\right) \mathrm{d} \mu_{j}(S)
$$

where $\chi$ denotes the Euler-Poincaré characteristic, and $S_{x}$ the translation of the subspace $S$ using the vector $x$. This equals 1 for any ovoid; while for any $K=\cup_{i=1}^{p} K_{i}$ (for $K_{i} \in C(\mathcal{K})$ ) we have an inclusion-exclusion formula:

$$
\chi(K)=\sum_{i} \chi\left(K_{i}\right)-\sum_{i_{1}<i_{2}} \chi\left(K_{i_{1}} \cap K_{i_{2}}\right)+\cdots+(-1)^{p+1} \chi\left(K_{1} \cap \cdots \cap K_{p}\right) .
$$

In particular the right-hand side does not depend on the particular representation $K=\cup_{i=1}^{p} K_{i}$ [17]. An equivalent definition uses the (generalised) Steiner formula

$$
\int \chi(K \cap B(x, t)) \mathrm{d} x=\sum_{j=0}^{d}\left(\begin{array}{l}
d \\
j
\end{array}\right) W_{j}^{d}(K) t^{j} .
$$

The extension (4) is by no means the only possibility; another option is to require the original formula (3) to hold for all $K$ in the convex ring, resulting in a different extension. But the Euler-Poincaré extension (4) has a useful relationship to 'number' which is exploited in various applications of stochastic geometry; in the planar $(d=2)$ case $W_{2}^{2}(K) / \pi$ is equal to the number of components of $K$ minus the number of holes of $K$. (For an example of this in the theory of high-level excursions of random fields, see Adler [1, Chapter 4].) 
Yet another possibility (albeit computationally more involved) is the positive extension, defined via a different generalised Steiner formula

$$
\int n(K ; r ; x) \mathrm{d} x=\sum_{j=0}^{d}\left(\begin{array}{l}
d \\
j
\end{array}\right) \bar{W}_{j}^{d}(K) t^{j}
$$

where $n(K ; r ; x)$ is the number of projections $y \in K$ of $x$ (points in $K$ locally closest to $x$ ) lying within distance $r$ of $x$ (see Matheron [36, (4-7-8)] or Schneider [58]). A significant subtlety in this definition is that it is possible to have $n(K ; r ; x)>1$ for $x \in \operatorname{int}(K)$. The $\bar{W}_{j}^{d}(K)$ defined here satisfy

$$
\bar{W}_{j}^{d}\left(\bigcup_{i} K_{i}\right) \leq \sum_{i} \bar{W}_{j}^{d}\left(K_{i}\right)
$$

for any compact convex sets $K_{1}, \ldots, K_{n}$. However in the following we shall focus our attention mainly on the simpler and more intuitive additive extension.

It is important that $\bar{W}_{j}^{d}(\cdot)=W_{j}^{d}(\cdot)$ for $j=0,1$, so the two main extensions discussed above will agree for the basic cases of Lebesgue measure and surface area.

\section{Quermass-interaction point processes}

\subsection{Notation and framework}

Let $\delta$ be $\mathbb{R}^{d}$ or a compact subset, $\mu$ a finite non-atomic measure on $\delta$ and $v$ a probability measure on $C(\mathcal{K})$. In this section we will define new germ-grain models by their density with respect to a Poisson marked point process of intensity $\mu \otimes v$ on $\delta \times C(\mathcal{K})$. Realisations will be denoted by $\boldsymbol{y}=\left\{\left(x_{1}, K_{1}\right), \ldots,\left(x_{n}, K_{n}\right)\right\}(n \geq 0)$.

Definition 2.1. A quermass-interaction germ-grain model is absolutely continuous with respect to a Poisson marked point process on $\delta \times C(\mathcal{K})$ with intensity $\mu \otimes \nu$, and has density $p(\boldsymbol{y})$ given by

$$
p(\boldsymbol{y})=\alpha \beta^{n(\boldsymbol{y})} \exp \left[-\sum_{j=0}^{d} \gamma_{j} W_{j}^{d}\left(u_{\boldsymbol{y}}\right)\right]
$$

Here $\beta>0$ and $\gamma_{j} \in \mathbb{R}$ are model parameters, $W_{j}^{d}$ is the additive extension (4) of the $j$ th Minkowski functional, and $U_{y}$ is the set union $\bigcup_{i=1}^{n}\left(K_{i}+x_{i}\right)$.

Note that the random marks will not be independent under (7). In a variation on this definition we may replace the additive extension by the positive extension (5).

An important special case is that where the mark distribution $v$ of the reference process is degenerate. In that case, the quermass-interaction germ-grain model (7) can be viewed as an unmarked point process on $\delta$ and (7) is its density with respect to a Poisson point process on $\&$ with intensity measure $\mu$.

The models in Definition 2.1 can be used to define quermass-interaction random sets by setting

$$
X=u_{Y}
$$


where $Y$ is a quermass-interaction germ-grain model. As in the Boolean model, the component grains are not observable since their boundaries may be occluded by other grains. Indeed, there is no way of determining even the number of germs giving rise to the union set $X$, complicating the question of estimation of model parameters (see later).

Special cases of Definition 2.1 have been discussed in the literature. The penetrable spheres $[21,54,55,65]$ or area-interaction model [4] is the case

$$
p(\boldsymbol{y})=\alpha \beta^{n(\boldsymbol{y})} \exp \left[-\gamma W_{0}^{d}\left(\boldsymbol{U}_{\boldsymbol{y}}\right)\right]
$$

and $v$ degenerate on a closed ball of radius $t>0$. Generalisations allow for the replacement of $W_{0}^{d}$ by any finite Borel regular measure. Note that even though the perimeter $W_{1}^{d}$ is a positive functional it is not a measure, and so the corresponding quermass-interaction point process does not fall within the generalised area-interaction framework of [4].

The case of Euler-Poincaré interaction $p(y)=\alpha \beta^{n(y)} \exp \left[-\gamma W_{3}^{3}\left(u_{y}\right)\right]$ in three dimensions is discussed from a physics point of view in $[34,38]$, though no consideration is given there to questions of existence and stability.

Finally, note that we have taken the approach (a) outlined in Section 1.2, in specifying densities with respect to a reference process which is a Poisson marked point process using the product measure $\mu \otimes v$ as intensity. Alternatively, the more general approach (b) (Section 1.2) may be taken, but it would result in more cumbersome formulae.

Area-interaction models are Markov (see Definition 1.1) with respect to the overlapping object relationship [3]

$$
(u, K) \sim(v, L) \quad \text { if and only if } \quad(K+u) \cap(L+v) \neq \varnothing
$$

and satisfy Ruelle's stability condition for all values of $\gamma$. This condition requires that the energy $E(\cdot)=-\log (p(\cdot) / p(\varnothing))$ is bounded below by a linear bound in the number of points:

$$
E(\boldsymbol{y}) \geq-A-B n(\boldsymbol{y})
$$

for some positive $A, B$. Indeed, the area-interaction density with respect to a Poisson $(\beta)$ process (restricted to a bounded window, as is always the case here) is uniformly bounded.

Stability is a sufficient condition for a measurable density to be integrable and is important with respect to simulation and edge effects. Indeed Ruelle [56, Section 3.2] shows that, for (upper semi-continuous) pairwise interaction processes, unless the energy is stable the weight functional will diverge for a motion-invariant point process extending over the whole of space. Gates and Westcott [13] show that, even in a bounded window and conditional on the number of points, unstable point processes may yield problems in simulations (published examples are typically not yet in equilibrium, and results will be very sensitive to boundary conditions) and approximations of the partition function can be wrong by many orders of magnitude. See also [40].

Before we start to investigate quermass-interaction point processes we must first show that they are well-defined, and the first step is the tedious but elementary chore of establishing the measurability of the function $p(\boldsymbol{y})$.

Lemma 2.2. The function $p(\boldsymbol{y})$ of Definition 2.1 is measurable with respect to $\mathcal{N}^{f}$, the Borel $\sigma$-algebra corresponding to the weak topology on the space of all integer-valued simple finite measures $\mathfrak{N}^{f}$. 
Proof. It suffices to consider $W_{j}^{d}\left(\mathcal{U}_{\boldsymbol{y}}\right)$ for all $j$. Using the inclusion-exclusion formula for $W_{j}^{d}\left(\mathcal{U}_{y}\right)$ and the fact that the mapping $(A, B) \mapsto A \cap B$ is upper-semicontinuous (see Corollary 1 to Proposition 1-2-4 in [36]), the measurability follows.

The measurability of the positive extension $\bar{W}_{j}^{d}\left(u_{y}\right)$ of the quermass integrals follows from the corollary on page 126 of [36]. Hence the analogue of (7) for the positive extension is measurable as well.

In order to check that the derived random set model (8) is well-defined, we need to check that hitting events $\{X \cap K \neq \varnothing\}$ are measurable. To see this, observe that

$$
\{X \cap K \neq \varnothing\}=\left\{\boldsymbol{y}: n\left(\boldsymbol{y}_{L}\right)=0\right\}^{c}
$$

where

$$
L=\{(u, Z):(Z+u) \cap K \neq \varnothing\} .
$$

As the mapping $f: \& \times C\left(\mathcal{K}^{\prime}\right) \rightarrow C\left(\mathcal{K}^{\prime}\right):(u, Z) \mapsto Z+u$ is continuous, $L$ is closed. Thus $\{X \cap K \neq \varnothing\}$ is measurable when viewed as a subset of $\left\{\mathfrak{N}^{f}, \mathcal{N}^{f}\right\}$, hence measurable when considered as an event.

We turn to the issue of when the quermass-interaction density is integrable, which is the main question addressed by this paper.

Note that the energy is

$$
-n(\boldsymbol{y}) \log \beta+\sum_{j=0}^{d} \gamma_{j} W_{j}^{d}\left(u_{\boldsymbol{y}}\right) .
$$

The stability condition for $\gamma>0$ would follow from

$$
W_{j}^{d}\left(u_{y}\right) \geq-B n(y)
$$

for each $j=0, \ldots, n$ whilst for $\gamma<1$ it would suffice to show

$$
W_{j}^{d}\left(u_{y}\right) \leq B n(y)
$$

(for some $B>0$ ). Since the $\log \beta$ term is linear in $n(y)$, it will not affect questions of stability.

We note in passing that the positive extension of Minkowski functionals always produces stability.

Lemma 2.3. Assume that in Definition 2.1 the positive extensions $\bar{W}_{j}^{d}(\cdot)$ of the Minkowski functionals are used. Then a quermass-interaction germ-grain model with $\gamma_{j} \geq 0$ $(j=1, \ldots, n)$ is stable. If $\gamma_{j}<0$, but $\bar{W}_{j}^{d}(K)$ is bounded above for all $K$ in the support of $v$, then the quermass-interaction germ-grain model is also stable.

Proof. It is sufficient to consider each $j=1, \ldots, n$ separately. For $\gamma_{j} \geq 0$ the stability inequality (9) is trivially verified. For $\gamma_{j}<0$, use subadditivity as given in (6): if $\boldsymbol{y}=\left\{\left(x_{1}, K_{1}\right), \ldots,\left(x_{n}, K_{n}\right)\right\}$ then

$$
0 \leq \bar{W}_{j}^{d}\left(u_{\boldsymbol{y}}\right) \leq \sum_{i=1}^{n} \bar{W}_{j}^{d}\left(K_{i}+x_{i}\right) .
$$


In fact even more can be said for the perimeter interaction germ-grain model $p(\boldsymbol{y})=\alpha \beta^{n(\boldsymbol{y})} \exp \left[-\gamma W_{1}^{d}\left(\boldsymbol{U}_{\boldsymbol{y}}\right)\right]$ when the grains are disks of constant radius. Stability follows as a consequence of [2], which actually proves the stronger result of a uniform bound on the density with respect to a Poisson process over a compact region. Complementary geometric arguments yield a uniform bound on the Papangelou conditional intensity [29].

Corollary 2.4. The quermass-interaction germ-grain model (Definition 2.1) using the positive extension is integrable whenever $\gamma_{j} \geq 0$ for all $j$; if on the other hand $\gamma_{j}<0$ then the density is still integrable provided that $\bar{W}_{j}^{d}(K)$ is uniformly bounded above for all $K$ in the support of $v$.

The situation is much more interesting for the additive extensions (except for $W_{0}^{d}(\cdot), W_{1}^{d}(\cdot)$ in which case the positive and additive extensions are identical). We shall focus on the planar Euler-Poincaré characteristic $W_{2}^{2}(\cdot)$. Further interest is added to this case because the density depends only on the topology of the union of the grains, noting that $W_{2}^{2}(K) / \pi$ equals the number of components of $K$ minus the number of holes of $K$. Clearly $n(\boldsymbol{y})$ provides an upper bound, hence the associated 'repulsive' quermass-interaction germ-grain model is stable. For the 'attractive' counterpart $\gamma_{2}>0$, we need an upper bound on the number of holes. This problem is dependent on the geometry of the grains and is treated in Sections 3, 4, 5.

\subsection{Markov properties}

As the area-interaction model [4], the quermass-interaction generalizations are Markovian in the sense of Definition 1.1.

Theorem 2.5. Whenever $p(\cdot)$ in Definition 2.1 is integrable, it is Markov with respect to the overlapping objects relation.

Proof. Property (M1) of the definition of a Markov point process is trivial since $p(\cdot)>0$, so it suffices to establish property (M2).

The case $W_{0}^{d}(\cdot)$ has been established in [4], so we consider $W_{j}^{d}(\cdot)$ for $j>0$. By the inclusion-exclusion formula,

$$
\begin{aligned}
-\log \frac{p(\boldsymbol{y} \cup\{(u, K)\})}{p(\boldsymbol{y})} & =\left[W_{j}^{d}\left(\mathcal{U}_{\boldsymbol{y}} \cup(K+u)\right)-W_{j}^{d}\left(\mathcal{u}_{\boldsymbol{y}}\right)\right] \gamma_{j}-\log \frac{1}{\beta} \\
& =\left[W_{j}^{d}((K+u))-W_{j}^{d}\left((K+u) \cap \mathcal{u}_{\boldsymbol{y}}\right)\right] \gamma_{j}-\log \frac{1}{\beta} \\
& =\left[W_{j}^{d}((K+u))-W_{j}^{d}\left((K+u) \cap \bigcup_{\left(x_{i}, K_{i}\right) \sim(u, K)}\left(K_{i}+x_{i}\right)\right)\right] \gamma_{j} \\
-\log \frac{1}{\beta} . &
\end{aligned}
$$

Thus the Papangelou conditional intensity for adding $(u, K)$ to $\boldsymbol{y}$ depends only on the subconfiguration of points $\left(x_{i}, K_{i}\right) \sim(u, K)$. Hence (M2) follows. There is a corresponding and straightforward argument for the positive extension, depending on the fact that for $x_{i}$ for which $\left(K_{i}+x_{i}\right) \cap(K+u)=\varnothing$ the exposed boundary in $\boldsymbol{y} \cup\{(u, K)\}$ is the same as in $\boldsymbol{y}$, so that a similar cancellation occurs. 
If it can be shown that the grain is always contained in a disk centred on $\mathbf{o}$ of fixed radius $r$ then the above argument establishes a local Markov property with respect to the conventional neighbourhood relationship $u \sim v$ when $u, v$ are closer than $2 r$.

A fortiori the process is nearest-neighbour Markov with respect to the connected component relation of Baddeley and Møller [5]. Note also the continuum random cluster model [19, $32,40]$, in which the weighting is carried out by counting connected components instead of calculating with the Euler characteristic. In that model the Markov property is replaced by a nearest-neighbour Markov property.

By the Hammersley-Clifford theorem (see Section 1.1 above), the density $p(\cdot)$ can be written as a product of clique interaction terms

$$
p(\boldsymbol{y})=\prod_{z \subseteq \boldsymbol{y}} q(\boldsymbol{z})
$$

where $q(z)=1$ unless $\left(z_{i}, L_{i}\right) \sim\left(z_{j}, L_{j}\right)$ for all elements of $z$. The interaction functions resemble those of the area-interaction model. For the additively extended $W_{j}^{d}$,

$$
\begin{aligned}
q(\varnothing) & =\alpha \\
q(\{(u, K)\}) & =\beta \gamma^{-W_{j}^{d}(K+u)} \\
q\left(\left\{\left(x_{1}, K_{1}\right), \ldots,\left(x_{k}, K_{k}\right)\right\}\right) & =\gamma^{(-1)^{k} W_{j}^{d}\left(\bigcap_{i=1}^{k}\left(K_{i}+x_{i}\right)\right)} ;
\end{aligned}
$$

(for positive extensions one can replace $W_{j}^{d}(K+u)$ by a boundary integral). In particular, the model has interaction of all orders.

The quermass-interaction germ-grain models $Y$ satisfy a spatial Markov property $[27,53]$

$$
Y \cap E \perp Y \cap D(E)^{c} \mid Y \cap D(E) \backslash E
$$

where $D(E)$ is the set of marked points that are related under $\sim$ to a marked point in $E$. In words, the random point pattern $Y \cap E$ is independent of the random point pattern $Y \cap D(E)^{c}$ when conditioned on the realization of the 'frontier' pattern $Y \cap D(E) \backslash E$.

It is possible to derive a random set Markov property in the 1-dimensional case. For example in $\mathbb{R}^{d}$, Matheron defines two compact sets $K$ and $K^{\prime}$ as separated by another compact set $C \in \mathcal{K}$ if any line segment joining $x \in K$ with $x^{\prime} \in K^{\prime}$ hits $C$. Furthermore, the random set $X$ is said to be semi-Markovian if a conditional independence property similar to (11) holds for $X \cap E, X \cap F \in \mathcal{K}$ for any sets $E$ and $F$ separated by $G \in \mathcal{K}$ and the conditioning is on $X \cap G=\varnothing$. It is then easy to show that the one-dimensional quermass-interaction random sets are semi-Markov.

No such result can be expected in higher dimensions, as separation no longer implies topological separation.

In the discrete case (grains replaced by pixels), Møller and Waagepetersen [41] have studied Markov connected component fields, and proved a characterization theorem. In particular, if the process is both a second order Markov random field and connected component field, the density factorises in terms related to area, perimeter and Euler characteristic, as well as continuity terms related to the digitization. 


\section{Planar case: illustrative examples}

In this section we consider in detail the two-dimensional case $d=2$ of additive quermassinteraction point processes in the plane. In this case, for $K \in C(\mathcal{K})$, we have

$$
\begin{aligned}
& W_{0}^{2}(K)=v_{2}(K)=\text { area } \\
& W_{1}^{2}(K)=\frac{1}{2} U(K)=\frac{1}{2} \text { perimeter }=\frac{\pi}{2} \text { mean breadth } \\
& W_{2}^{2}(K)=\pi
\end{aligned}
$$

and we study the point process whose density with respect to a Poisson point process (or marked Poisson point process, if the grains are random) is given by

$$
p(\boldsymbol{y})=\alpha \beta^{n(\boldsymbol{y})} \gamma_{0}^{-W_{0}^{d}\left(u_{y}\right)} \times \cdots \times \gamma_{d}^{-W_{d}^{d}\left(u_{y}\right)} .
$$

In the instances $r=0$ and $r=1$, the functional $W_{r}^{2}(\cdot)$ is positive, both extension methods coincide, and Lemma 2.3 leads to the simple conclusion that integrability holds for all values of $\gamma$ (at least for bounded convex grains as above). So it remains to consider the case $r=2$.

Here the extension methods do not coincide and we have to argue in detail. The additive extension of $W_{2}^{2}(\cdot)$ has a simple interpretation: it is proportional to the Euler-Poincaré characteristic $\chi$ ('the number of components minus the number of holes' for this planar case). In fact $W_{2}^{2}(K)=\pi \chi(K)$. For Ruelle stability (9) to hold for all parameter values, we require

$$
-B_{1} n(y) \leq \chi\left(\mathcal{U}_{\boldsymbol{y}}\right) \leq B_{2} n(\boldsymbol{y})
$$

where $B_{1}, B_{2}$ are positive constants. The right-hand inequality is immediate from $C$-additivity, but the left-hand inequality is actually false in general (see the illustrative examples below). Let us examine what can go amiss. First note that if $\gamma=1$ then the weighting has no effect and everything is trivial. The case $\gamma<1$ (inhibition) is also clear:

$$
\int \ldots \int \gamma^{-\pi \chi\left(u_{y}\right)} \mathrm{d} \mu\left(x_{1}\right) \mathrm{d} \nu\left(K_{1}\right) \ldots \mathrm{d} \mu\left(x_{n}\right) \mathrm{d} \nu\left(K_{n}\right) \leq \gamma^{-\pi n(\boldsymbol{y})} \mu(\mathcal{X})^{n(\boldsymbol{y})} .
$$

and so the process is then bounded above by a Poisson process with intensity measure $\gamma^{-\pi} \mu(\cdot)$ and therefore is integrable and indeed stable. However stability does not hold in general for the clustered case of $\gamma>1$, as we now indicate by exhibiting various illustrative examples.

The first illustrative example is suggested by the observation that $n$ lines in general position in the plane produce $(n-1)(n-2) / 2$ (bounded) holes. (Recall that 'general position' means that no three lines meet at one point.) The proof is by induction: adding a line in general position to an assembly of $n$ lines in general position produces $n-1$ new bounded holes.

Example 3.1 (Poisson line process in the plane.) Let the germ process be an inhomogeneous Poisson point process of finite total intensity. Let the typical grain be a line randomly oriented with some fixed directional distribution. Suppose that the intensity measure of the resulting line process is diffuse and has topological support containing two lines which intersect (of course this second requirement will be fulfilled unless all lines in the process are almost surely parallel!). Then the expectation with respect to the Poisson line process

$$
\mathbb{E}\left\{\gamma^{-W_{2}^{2}\left(u_{y}\right)}\right\}=\mathbb{E}\left\{\exp \left(-(\ln \gamma) \times W_{2}^{2}\left(u_{y}\right)\right)\right\}
$$

is infinite if $\gamma>1$. 
Since $W_{2}^{2}\left(U_{y}\right) / \pi=\chi\left(U_{y}\right)$ is 'the number of components minus the number of holes' this means that we cannot weight the model towards having more holes than would be expected in the unweighted (Poisson) case.

Proof. Note that $-\chi\left(\mathcal{U}_{y}\right)$ is bounded above by the number of (bounded) holes in $\mathcal{U}_{\boldsymbol{y}}$. The topological support condition means that we can choose two compact sets $K_{1}, K_{2}$ in line space such that $(a)$ the intensity measure charges both $K_{1}$ and $K_{2}$, and $(b)$ all lines in $K_{1}$ intersect all lines in $K_{2}$. We condition on the event that all lines of the process belong to $K_{1} \cup K_{2}$. Under this conditioning event (which is of positive probability) the number of holes is given by $\left(N_{1}-1\right)\left(N_{2}-1\right) / 2$ where $N_{i}$ is the (random) number of lines in $K_{i}$ and has a nondegenerate Poisson distribution. But this means that the expectation in Equation (13) is infinite, because the moment generating function of the product of two non-degenerate Poisson distributions is infinite for positive argument.

It might be objected that the above example uses non-compact grains of zero area, so that the grains are definitely not ovoids. Basic arguments using Boolean models readily yield the following localization and conditioning argument which replaces unbounded lines by bounded line segments: it is then a straightforward if tedious exercise to make further modifications to produce a genuine counterexample based on thin random rectangles (we can supply details on request).

Example 3.2 (Poisson segment process in the plane.) Let the germ process be an inhomogeneous Poisson point process of finite total intensity. Let the typical grain be a line segment randomly oriented with some fixed directional distribution. Suppose that the intensity measure of the resulting line segment process is diffuse and has topological support containing two line segments which intersect. Then the expectation with respect to the Poisson segment process

$$
\mathbb{E}\left\{\gamma^{-W_{2}^{2}\left(u_{y}\right)}\right\}=\mathbb{E}\left\{\exp \left(-(\ln \gamma) \times W_{2}^{2}\left(u_{y}\right)\right)\right\}
$$

is infinite if $\gamma>1$.

Proof. We can argue exactly as in Example 3.1, except that this time the topological support condition allows us to choose compact sets in segment space, $K_{1}$ and $K_{2}$, such that (a) the intensity measure charges both $K_{1}$ and $K_{2}$, and $(b)$ each segment in $K_{1}$ intersects all segments in $K_{2}$.

The problems in the above two examples appear to be related to the pathological 'sharpness' of the grains, and in particular to the fact that they have negligible area. A natural condition to exclude this pathology is to require a lower bound on the internal angles of convex polygonal grains.

Definition 3.3. A convex grain $G$ is said to satisfy a 'local wedge condition of angle $\phi>0$ ' if for any point $\omega \in \partial G$ there is a disk $B(\omega, r)$ (centred at $\omega$, of positive radius $r=r(\omega)$ ) such that $B(\omega, r) \cap G$ is a sector of the disk of angle at least $\phi$. (No lower bound is placed on the radius of the disk, other than the requirement that it be positive.)

Note that a convex grain satisfying this condition is automatically polygonal.

Here is a counterexample to show that care is required even when the grains satisfy a local wedge condition (note that another counterexample is provided by the thin rectangle modification alluded to in the discussion of Example 3.2). 


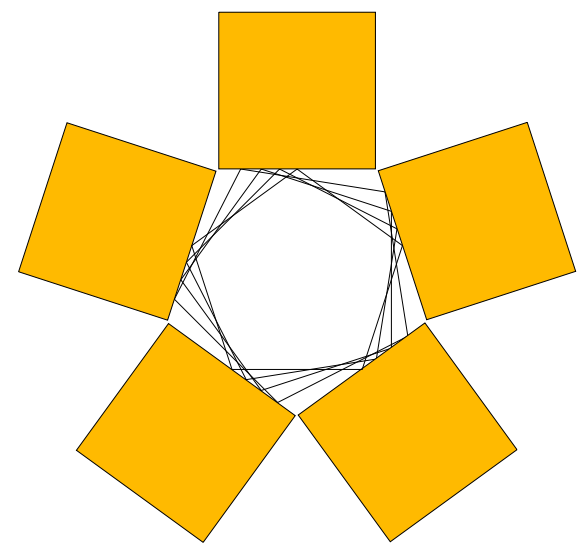

FIGURE 1: How to build configurations of $n$ polygonal grains which create $O\left(n^{2}\right)$ holes (pentagonal case). We can arrange $k=n / 2$ pentagons one on top of the other so that by adding $r=n / 2$ other polygons we can create $n / 4$ holes per polygon.

Example 3.4 (Germ-grain model with ovoid grains satisfying a local wedge condition.) In general the weighting need not satisfy the stability condition when $\gamma>1$.

Proof. The grains are regular $k$-gons, of varying side-number $k(k>3)$ and size. To establish failure of stability, we have to show how to construct configurations of $n$ grains which possess $O\left(n^{2}\right)$ holes.

Fix $k>3, r>1$ and set $\epsilon=(r-1) \pi /\left(k(k-1)^{2}\right)$. Notice that the local wedge condition is satisfied for $\phi=\pi / 2$, since $k>3$.

Consider $r$ similar $k$-gons, of which the first is inscribed in a circle of unit radius centered on the origin $\boldsymbol{o}$, and such that the $i$ th $k$-gon is obtained from the first by rotation about $\boldsymbol{o}$ through an angle of $(i-1) \epsilon$ and scaling (again about $\boldsymbol{o})$ by a factor of $\sec ((i-1) \epsilon)$. At each vertex of the first $k$-gon place a square with sides of unit length, tangent to the inscribing circle at the midpoint of a side. (See Figure 1 for the case $k=5, r=5$.)

For all sufficiently large $k$, each square intersects each of the $k$-gons at a vertex, and none of the intersections of squares with $k$-gons are covered by other squares or $k$-gons. (This follows from the observation that the $k$-gons intersect in singleton sets with lines through vertices of the first polygon which are perpendicular to radii of the circle which it inscribes.) Consequently this configuration of $(r-1) k$-gons and $k$ squares creates at least $k(r-1)$ holes. Setting $k=r=n / 2$ for even $n$ delivers the required violation of stability.

It is important to note that the above counterexample works only if we allow polygonal grains of arbitrarily small sidelength. Later on we shall see that an additional lower bound on sidelength (obtained by requiring a uniform local wedge condition) is sufficient to ensure stability for polygonal grains.

In this paper we confine ourselves to the planar case, which is the case of principal importance for image analysis (though not for physics! see [34, 37, 38]). However it is interesting to note that things can go even more badly wrong for the Euler-Poincaré characteristic in the spatial case. We illustrate this with a simple non-ovoid example (as before, it is a straightforward but tedious exercise to modify this to produce a counterexample using ovoids). 
Example 3.5 (Process of flats in space.) Divergence can occur for all parameter values except for the trivial (unweighted) case of $\gamma=1$. Take the Poisson point process of germs to be inhomogeneous and of unit total intensity. Fix an orthonormal basis. Let the typical grain be a 'flat' or 2-plane, normal to a vector chosen randomly from the orthonormal basis with probabilities $\lambda_{1}, \lambda_{2}, \lambda_{3}$. Suppose that the intensity measure of the underlying flat process is diffuse. Then the expectation with respect to the Poisson flat process

$$
\mathbb{E}\left\{\gamma^{-W_{3}^{3}\left(u_{y}\right)}\right\}=\mathbb{E}\left\{\exp \left(-(\ln \gamma) \times W_{3}^{3}\left(u_{y}\right)\right)\right\}
$$

is finite if and only if $\gamma=1$ (the trivial unweighted case!).

Proof. First note that $W_{3}^{3}\left(u_{y}\right)$ is no longer proportional to the number of holes minus the number of components, but is proportional to the three-dimensional Euler-Poincare characteristic. However (in the simple case which we have chosen to consider) it is easily computed from first principles using the inclusion-exclusion formula of $C$-additivity. Let $N_{1}, N_{2}, N_{3}$ be the numbers of flats normal to each of the three basis vectors. Then

$$
\begin{aligned}
W_{3}^{3}\left(\boldsymbol{U}_{\boldsymbol{y}}\right) & =\left(N_{1}+N_{2}+N_{3}\right)-\left(N_{1} N_{2}+N_{2} N_{3}+N_{3} N_{1}\right)+N_{1} N_{2} N_{3} \\
& =\left(N_{1}-1\right)\left(N_{2}-1\right)\left(N_{3}-1\right)+1
\end{aligned}
$$

(since intersections of more than three flats will be almost surely void, because the underlying intensity measure of the flat process is diffuse). It suffices to show divergence of $\mathbb{E}\left\{\gamma^{-\left(N_{1}-1\right)\left(N_{2}-1\right)\left(N_{3}-1\right)-1}\right\}$.

Suppose that $\gamma<1$. Then (noting that $\lambda_{1}+\lambda_{2}+\lambda_{3}=1$ )

$$
\begin{aligned}
\mathbb{E}\left\{\gamma^{-\left(N_{1}-1\right)\left(N_{2}-1\right)\left(N_{3}-1\right)-1}\right\} & =\sum_{n_{1}} \sum_{n_{2}} \sum_{n_{3}} \mathrm{e}^{-1} \frac{\lambda_{1}^{n_{1}} \lambda_{2}^{n_{2}} \lambda_{3}^{n_{3}}}{n_{1} ! n_{2} ! n_{3} !} \gamma^{-\left(n_{1}-1\right)\left(n_{2}-1\right)\left(n_{3}-1\right)-1} \\
& \geq \sum_{n} \mathrm{e}^{-1} \frac{\lambda_{1}^{n} \lambda_{2}^{n} \lambda_{3}^{n}}{n !^{3}} \gamma^{-(n-1)^{3}-1}=\infty
\end{aligned}
$$

where the divergence follows from Stirling's formula.

Suppose $\gamma>1$. Then consider the bound obtained by restricting the above expectation to the event, which is of positive probability, that $N_{3}=0$. We have

$$
\begin{aligned}
\mathbb{E}\left\{\gamma^{-\left(N_{1}-1\right)\left(N_{2}-1\right)\left(N_{3}-1\right)-1}\right\} & \geq \mathbb{E}\left\{\gamma^{-\left(N_{1}-1\right)\left(N_{2}-1\right)\left(N_{3}-1\right)-1} \mid N_{3}=0\right\} \times \mathbb{P}\left\{N_{3}=0\right\} \\
& =\sum_{n_{1}} \sum_{n_{2}} \mathrm{e}^{-1} \frac{\lambda_{1}^{n_{1}} \lambda_{2}^{n_{2}}}{n_{1} ! n_{2} !} \gamma^{\left(n_{1}-1\right)\left(n_{2}-1\right)-1} \\
& \geq \sum_{n} \mathrm{e}^{-1} \frac{\lambda_{1}^{n} \lambda_{2}^{n}}{n !^{2}} \gamma^{(n-1)^{2}-1}=\infty
\end{aligned}
$$

where once again the divergence follows from Stirling's formula.

Naiman and Wynn have generously contributed the following counterexample, which shows that in 4-space one cannot expect convergence for all parameter values even in the wellbehaved case of balls of unit radius.

Example 3.6 (Unit balls in 4-space.) Consider the germ-grain model $u_{\boldsymbol{y}}$ based on an inhomogeneous Poisson process of finite total intensity in 4-space with grains which are unit 


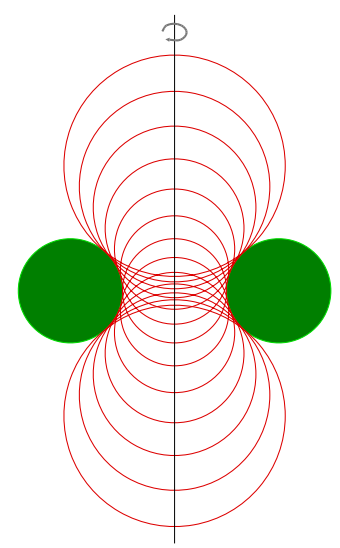

FIGURE 2: Vertical section view of $2 n$ balls of varying radius in 3-space, whose union has EulerPoincaré characteristic $1-n^{2}$. First arrange $n$ unit-radius balls in an overlapping horizontal ring (two of these seen in section as dark circles). Then build a fan of $n$ balls with centres located along the axis of symmetry, so that the balls in the fan form a connected union and each ball in the fan touches each of the first $n$ balls in one point only.

balls. Suppose that the intensity measure has a density which is constant over the ball centred on the origin and of radius $\sqrt{2}$. Then for $\gamma>1$ the distribution produced by weighting using

$$
\gamma^{-W_{4}^{4}\left(u_{y}\right)}
$$

is not stable in Ruelle's sense.

Proof. First note that $W_{4}^{4}\left(u_{y}\right)$ is proportional to the Euler-Poincaré characteristic of $\boldsymbol{u}_{\boldsymbol{y}}$.

Consider the ensemble of $2 n$ balls of unit radius, of which the first $n$ are centred respectively at $(\sqrt{2} \cos (2 k \pi / n), \sqrt{2} \sin (2 k \pi / n), 0,0)$ for $k=1, \ldots, n$, and the second $n$ are centred respectively at $(0,0, \sqrt{2} \cos (2 k \pi / n), \sqrt{2} \sin (2 k \pi / n))$ for $k=1, \ldots, n$. (The condition on the density of the intensity measure is imposed in order to ensure that such a configuration is feasible for $U_{y}$.) The first $n$ balls form a sub-ensemble whose union is homotopic to a circle (for large enough $n$ ) and therefore has Euler-Poincaré characteristic 0, and similarly for the sub-ensemble of the other $n$ balls.

However intersections between balls from the first and second sub-ensembles are pairwise only, and are singleton sets for every possible intersection of this kind. It follows from the inclusion-exclusion identity that the Euler-Poincare characteristic of the union of all $2 n$ balls is $-n^{2}$. Hence Ruelle stability fails.

It is an open question whether stability fails for the weighting $\gamma^{-W_{3}^{3}\left(U_{y}\right)}(\operatorname{case} \gamma \neq 1)$ when $u_{y}$ is the germ-grain model produced by using unit balls in 3-space. However Naiman and WSK have independently produced a counterexample for the case of balls of random radius in 3-space, based on Figure 4.7.1 from [49] (see also [31]). See Figure 2 for an indication of the construction.

At this point we note in passing the early work of Eckhoff [10], who discusses rather general bounds on the range of values of the Euler-Poincaré characteristic.

These examples show that even in the planar case some conditions are needed if the range of $\gamma$ is to be unconstrained. On the other hand the planar examples appear to be somewhat 
pathological. Note that the problems are local (the treatment of the line process case makes this clear) and appear in Examples 3.1, 3.2, 3.5 to be related to the 'sharpness' of the constituent grains, while Example 3.4 shows problems arise when grains of 'small' sidelength are allowed.

There are two positive results which cover an important range of practical examples, and which serve to clarify the sense in which the above examples are pathological. These cover the complementary cases of (a) random disk grains and (b) random polygon grains which are neither too sharp nor too small. We deal with these results in the two following sections.

As a final remark, note that it is natural to enquire whether the divergence (in $d=2$ at least) can ever occur if the grains are non-random. Divergence can occur for simple non-convex nonrandom grains: consider the case of a grain composed of intersecting horizontal and vertical line segments, and apply the ideas underlying Example 3.2. In the case of convex non-random grains which are polygons, one can argue that either the grains are parallel line segments or parallel lines (in which trivial case stability is immediate, as there will be no holes!) or they must satisfy a uniform local wedge condition (given below as Definition 5.1), in which case stability follows from the arguments in Section 5. The case of non-polygonal convex nonrandom grains is currently open, with the exception of grains which are disks, which case is covered by the results in the following section.

\section{Planar case: when grains are disks}

In this section we show that if the grains are random disks then the Euler-Poincaré quermass-interaction germ-grain model

$$
p(\boldsymbol{y})=\alpha \beta^{n(\boldsymbol{y})} \exp \left[-\gamma W_{2}^{2}\left(\boldsymbol{U}_{\boldsymbol{y}}\right)\right]
$$

is stable, and hence integrable for all values of the parameter $\gamma$. Remarkably, no size constraint is required: the disk radii can be random and need only be strictly positive. This is particularly striking in the light of the examples in the previous section, which suggest that stability problems arise when side length is small. Here we see such problems need not occur at the limit. The argument is strictly geometrical, and is to be found in the theorem below: an ensemble of $N$ disks has a union with at most $2 N-5$ holes.

If the disks are of constant size then there is an easy argument using the Dirichlet tessellation based on the disk centres: we sketch it here. Let $B\left(x_{1}, r_{1}\right), B\left(x_{2}, r_{2}\right), \ldots, B\left(x_{N}, r_{N}\right)$ be the (closed) disks. In each component of the complement of $\bigcup_{i} B\left(x_{i}, r_{i}\right)$ there must be at least one node of the tessellation (a node is a vertex of the planar linear graph formed by the tessellation, including the 'vertex at infinity'), for otherwise the boundary of this component would have to be made out of the boundaries of at most two disks (which would force the 'vertex at infinity' to belong to the complement). Hence the number of holes in the union $\bigcup_{i} B\left(x_{i}, r_{i}\right)$ is dominated by the number of nodes of the Dirichlet tessellation, equivalently the number of triangles in the Delaunay tessellation, which by planar graph theory (using the Euler formula; see for example [66, Theorem 13A]) is itself dominated by the bound $2 N-5$, since $N$ is the number of vertices of the Delaunay tessellation (note the bound is not $2 N-6$, as we exclude the hole at infinity).

Unfortunately this simple argument appears not to generalize, being tied to the Euclidean metric structure underlying the definitions of a disk and of Dirichlet and Delaunay tessellations. For disks of arbitrary radius we have to argue carefully about how to reduce the union of disks to a planar network without decreasing the number of holes. The reduction uses line segments connecting certain of the disk centres (together with some polygons): the main technical issue is to choose a set of such line segments which leave connectivity unchanged and which do not 


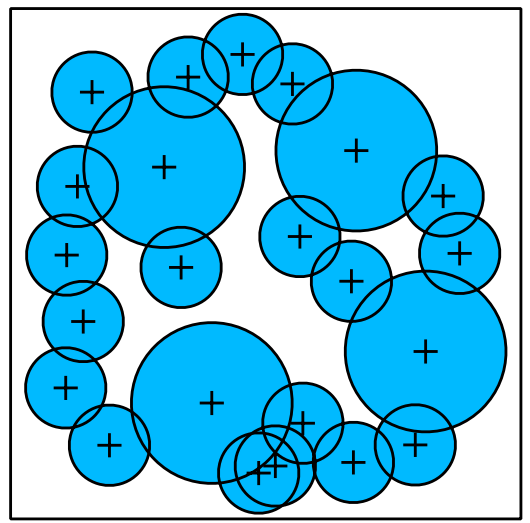

FIGURE 3: A typical field of overlapping disks $B\left(x_{1}, r_{1}\right), B\left(x_{2}, r_{2}\right), \ldots, B\left(x_{N}, r_{N}\right)$ of varying sizes.

cross each other. Naiman and Wynn have recently discovered a delightful argument deriving Theorem 4.3 from their work on abstract tube theory [43, 45], based on an algebraic topology argument related to the Morse inequalities. However the argument given below is more selfcontained, and in particular avoids algebraic topology.

We commence by introducing notation and proving two preliminary lemmas.

Consider an ensemble $B\left(x_{1}, r_{1}\right), B\left(x_{2}, r_{2}\right), \ldots, B\left(x_{N}, r_{N}\right)$ of $N$ overlapping closed disks of varying sizes all lying in the plane. Set $\mathscr{D}$ to be the union of the disks, and $\mathscr{D}_{0}$ to be the union of the interiors of the disks, so that

$$
\begin{aligned}
\mathscr{D} & =\bigcup_{i=1}^{N} B\left(x_{i}, r_{i}\right) \\
\mathcal{D}_{0} & =\bigcup_{i=1}^{N} \operatorname{int}\left(B\left(x_{i}, r_{i}\right)\right) .
\end{aligned}
$$

We suppose that they are placed in general position, so that no more than two disk boundaries intersect at any given point, and so that if two disk boundaries do intersect then they intersect at two distinct points. Figure 3 illustrates a possible arrangement: close inspection will reveal that the disks here are in fact in general position!

Each pair of overlapping disks $B\left(x_{i}, r_{i}\right), B\left(x_{j}, r_{j}\right)$ has boundaries intersecting in two points $x_{i j}^{-}, x_{i j}^{+}$, where the order of $i$ and $j$ and the sign are chosen by an arbitrary convention so that $i<j$ and $x_{i j}^{+}$is on the clockwise side of $B\left(x_{i}, r_{i}\right) \cap B\left(x_{j}, r_{j}\right)$ when viewed from the centre of $B\left(x_{i}, r_{i}\right)$.

For each point of intersection $x_{i j}^{ \pm}$of the boundaries of two disks $B\left(x_{i}, r_{i}\right), B\left(x_{j}, r_{j}\right)$, if $x_{i j}^{ \pm}$ is not covered by $\mathscr{D}_{0}$ then define $T_{i j}^{ \pm}$to be the closed triangular region with vertices at $x_{i j}^{ \pm}$ and the centres of $B\left(x_{i}, r_{i}\right), B\left(x_{j}, r_{j}\right)$. Define $S_{i j}$ to be the line segment running between the centres of $B\left(x_{i}, r_{i}\right), B\left(x_{j}, r_{j}\right)$.

We say that $T_{i j}^{ \pm}$is not defined if the corresponding $x_{i j}^{ \pm}$is covered by $\mathscr{D}_{0}$. We say that $S_{i j}$ is not defined if both the corresponding $x_{i j}^{+}$and $x_{i j}^{-}$are covered by $\mathscr{D}_{0}$. 


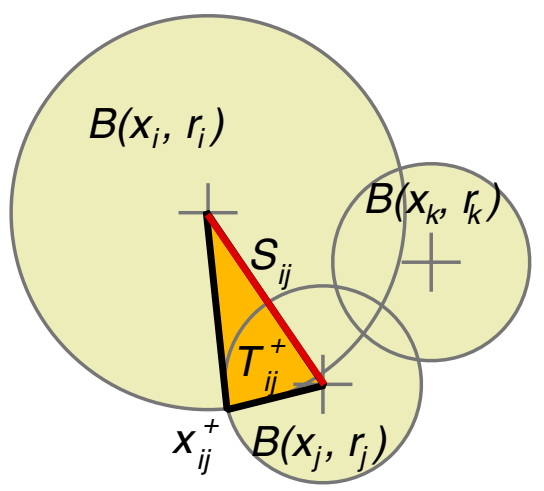

FIGURE 4: The closed triangular region $T_{i j}^{+}$with vertices at $x_{i j}^{+}$and the centres of $B\left(x_{i}, r_{i}\right), B\left(x_{j}, r_{j}\right)$. In this example $T_{i j}^{-}$is not defined, since $x_{i j}^{-}$is covered by the interior of a third disk $B\left(x_{k}, r_{k}\right)$.

Figure 4 illustrates the definition of $x_{i j}^{+}, T_{i j}^{+}$and $S_{i j}$. Note that if $T_{i j}^{+}$(respectively $T_{i j}^{-}$) is defined then $x_{i j}^{+}$(respectively $x_{i j}^{-}$) is a 'corner' of the union $\mathscr{D}$. We now make some observations about these triangular regions. Firstly we note that they serve as 'dead areas' for disks, in the sense that if a $B\left(x_{k}, r_{k}\right)$ has centre $x_{k}$ lying in $T_{i j}^{ \pm}$(for $i, j, k$ distinct) then it cannot contribute any exposed $x_{k \ell}$. This follows readily from geometric intuition, but here we give a rigorous proof based on homogeneous coordinates.

Lemma 4.1. If $T_{i j}^{+}$(respectively $T_{i j}^{-}$) is defined then any further disk $B\left(x_{k}, r_{k}\right)$ with centre in $T_{i j}^{+}$(respectively $\left.T_{i j}^{-}\right)$must be wholly contained in $\operatorname{int}\left(B\left(x_{i}, r_{i}\right)\right) \cup \operatorname{int}\left(B\left(x_{j}, r_{j}\right)\right)$.

Proof. Without loss of generality consider $T_{i j}^{+}$. Because $T_{i j}^{+}$is defined, $x_{i j}^{+}$must lie outside $B\left(x_{k}, r_{k}\right)$ (recall that the disks are placed in general position, so we can replace $\operatorname{int}\left(B\left(x_{k}, r_{k}\right)\right.$ ) by $B\left(x_{k}, r_{k}\right)$ here). (Figure 5 illustrates the situation.)

Choose coordinates such that $x_{i j}^{+}=0$ and the centres of $B\left(x_{i}, r_{i}\right), B\left(x_{j}, r_{j}\right)$ are at $a, b$ respectively. If the centre of $B\left(x_{k}, r_{k}\right)$ lies in $T_{i j}^{+}$then it is at $\lambda a+\mu b$, for $\lambda+\mu \leq 1, \lambda \geq 0$, $\mu \geq 0$. (In fact $\lambda, \mu, 1-\lambda-\mu$ provide a system of homogeneous coordinates for the centre of $B\left(x_{k}, r_{k}\right)$.)

Consider a point $y$ lying outside the interiors of both $B\left(x_{i}, r_{i}\right)$ and $B\left(x_{j}, r_{j}\right)$. This means

$$
\begin{gathered}
\|y-a\| \geq\|a\|, \\
\|y-b\| \geq\|b\|,
\end{gathered}
$$

and on squaring and simplifying we find

$$
\begin{gathered}
\|y\|^{2}-2\langle y, a\rangle \geq 0, \\
\|y\|^{2}-2\langle y, b\rangle \geq 0 .
\end{gathered}
$$

Hence we deduce

$$
(\lambda+\mu)\|y\|^{2}-2\langle y,(\lambda a+\mu b)\rangle \geq 0
$$

(note that $\lambda$ and $\mu$ are both non-negative!) and therefore, because $\lambda+\mu \leq 1$,

$$
\|y-(\lambda a+\mu b)\| \geq\|\lambda a+\mu b\| .
$$




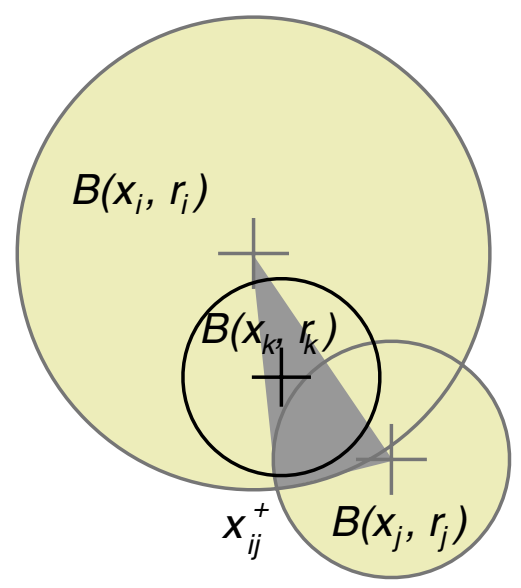

FIGURE 5: An argument using homogeneous coordinates, based on $0=x_{i j}^{+}$and the centres of $B\left(x_{i}, r_{i}\right)$ and $B\left(x_{j}, r_{j}\right)$, shows that if $T_{i j}^{+}$is defined, and if $B\left(x_{k}, r_{k}\right)$ is centred in $T_{i j}^{+}$, then $B\left(x_{k}, r_{k}\right)$ is contained in the union of the interiors of $B\left(x_{i}, r_{i}\right)$ and $B\left(x_{j}, r_{j}\right)$.

But $B\left(x_{k}, r_{k}\right)$ must not contain $x_{i j}^{+}$, and this means that its radius must be strictly less than $\|\lambda a+\mu b\|$. So $y$ must lie outside $B\left(x_{k}, r_{k}\right)$, and so $B\left(x_{k}, r_{k}\right)$ must be contained in $\operatorname{int}\left(B\left(x_{i}, r_{i}\right)\right) \cup \operatorname{int}\left(B\left(x_{j}, r_{j}\right)\right)$.

Therefore no $T_{k \ell}^{+}$or $S_{k \ell}$ can be defined for such a $B\left(x_{k}, r_{k}\right) ; T_{i j}^{+}$is a 'dead area' for disks. A similar argument holds for $T_{i j}^{-}$.

Secondly we note that no two of these 'dead-area' triangles can have overlapping interiors.

Lemma 4.2. No two triangles $T_{i j}^{ \pm}, T_{r s}^{ \pm}$can have overlapping interiors. (Here the \pm superscript refers systematically to one of + or - in each of the two cases of $T_{i j}^{ \pm}, T_{r s}^{ \pm}$).

Proof. Let $x_{i}, x_{j}, x_{r}, x_{s}$ be the centres of disks $B\left(x_{i}, r_{i}\right), B\left(x_{j}, r_{j}\right), B\left(x_{r}, r_{r}\right), B\left(x_{s}, r_{s}\right)$ respectively. Let $x_{i j}^{ \pm}, x_{r s}^{ \pm}$be exposed intersections of the respective disk boundaries. Suppose that a point $u$ is in the interiors of both the triangle $x_{i} x_{j} x_{i j}^{ \pm}$and the triangle $x_{r} x_{s} x_{r s}^{ \pm}$. We derive a contradiction from this and the requirement of the disks being in general position, as follows.

First observe that by the previous lemma we can deduce that the open disk $\tilde{D}$ of centre $u$ and radius $\left|u-x_{i j}^{ \pm}\right|$is contained in $\operatorname{int}\left(B\left(x_{i}, r_{i}\right)\right) \cup \operatorname{int}\left(B\left(x_{j}, r_{j}\right)\right)$. Thus we can add a further closed disk $B\left(x_{N+1}, r_{N+1}\right)$ to the original assembly of disks $B\left(x_{1}, r_{1}\right), B\left(x_{2}, r_{2}\right), \ldots, B\left(x_{N}, r_{N}\right)$ without altering the union of all the disks, where $B\left(x_{N+1}, r_{N+1}\right)$ is a closed disk of centre $u$ and radius less than but arbitrarily close to $\left|u-x_{i j}^{ \pm}\right|$. Consequently $B\left(x_{N+1}, r_{N+1}\right)$ cannot cover $x_{r s}^{ \pm}$, since otherwise $x_{r s}^{ \pm}$would be covered by $\mathscr{D}_{0}=\bigcup_{i=1}^{N} \operatorname{int}\left(B\left(x_{i}, r_{i}\right)\right)$, contradicting our assertion that $T_{r s}^{ \pm}$is defined.

Working with the new assembly $B\left(x_{1}, r_{1}\right), B\left(x_{2}, r_{2}\right), \ldots, B\left(x_{N}, r_{N}\right), B\left(x_{N+1}, r_{N+1}\right)$, we can also apply the previous lemma to $T_{r s}^{ \pm}$and $B\left(x_{N+1}, r_{N+1}\right)$, to deduce that

$$
B\left(x_{N+1}, r_{N+1}\right) \subseteq \operatorname{int}\left(B\left(x_{r}, r_{r}\right)\right) \cup \operatorname{int}\left(B\left(x_{s}, r_{s}\right)\right) .
$$

Since the radius of $B\left(x_{N+1}, r_{N+1}\right)$ is arbitrarily close to $\left|u-x_{i j}^{ \pm}\right|$, we deduce that $\tilde{D} \subset$ $\operatorname{int}\left(B\left(x_{r}, r_{r}\right)\right) \cup \operatorname{int}\left(B\left(x_{s}, r_{s}\right)\right)$. 

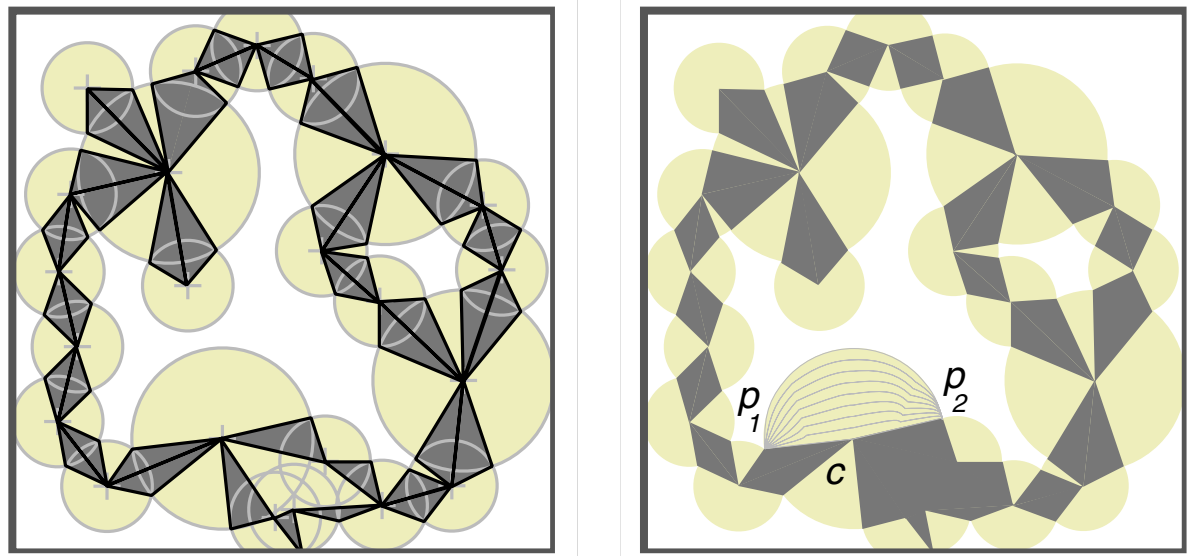

Figure 6: (a): $\mathscr{D}$ together with the configuration of defined triangles $T_{i j}^{ \pm}$. (b): Construction of $\tilde{D}$ from $\mathscr{D}$ by retracting the 'edges' back to joined pairs of triangle segments.

But now we have shown that the open disk $\tilde{D}$ of center $u$ and radius $\left|u-x_{i j}^{ \pm}\right|$is contained in $\operatorname{int}\left(B\left(x_{r}, r_{r}\right)\right) \cup \operatorname{int}\left(B\left(x_{s}, r_{s}\right)\right)$, while $x_{i j}^{ \pm}$is not so contained (since it is exposed). So $x_{i j}^{ \pm}$lies on the boundaries of $B\left(x_{r}, r_{r}\right), B\left(x_{s}, r_{s}\right)$, as well as on the boundaries of $B\left(x_{i}, r_{i}\right), B\left(x_{j}, r_{j}\right)$. At least three of these disks are distinct, so this violates the requirement for the disks to be in general position. We deduce that the interiors of the triangles $x_{i} x_{j} x_{i j}^{ \pm}$and $x_{r} x_{s} x_{r s}^{ \pm}$are disjoint, as required.

We now turn to the main result of this section.

Theorem 4.3. For $D$ a union of $N$ closed disks in the plane, the number of holes in $\mathscr{D}$ is bounded above by $2 \mathrm{~N}-5$.

Proof. We may suppose the disks are in general position as described at the beginning of this section. We use the notation established above.

For every (exposed) 'corner' $x_{i j}^{ \pm}$of $\mathscr{D}$ we have defined a 'dead-area' triangle $T_{i j}^{ \pm}$with vertices at $x_{i j}^{ \pm}$and the centres of the two disks $B\left(x_{i}, r_{i}\right), B\left(x_{j}, r_{j}\right)$ whose overlapping forms the 'corner'. Moreover we have shown that the interiors of distinct defined 'dead-area' triangles do not overlap. The resulting configuration of defined triangles $T_{i j}^{ \pm}$is shown in Figure 6(a).

The 'corners' of $\mathscr{D}$ divide the boundary $\partial \mathscr{D}$ into 'edges' (circular arcs). To each 'edge' we can associate two bounding 'corners', $p_{1}$ and $p_{2}$, except when the 'edge' is a complete circle, corresponding to a disk separated from all the others (note that the configuration of general position removes ambiguous cases). We need not consider the exceptional case, as this makes no contribution to the number of holes of $\mathscr{D}$. For the non-exceptional edges the corresponding triangles share a vertex which is a disk centre $c$. The non-overlapping property given in Lemma 4.2 means we can retract each 'edge' back to the joined segments $p_{1} \rightarrow c \rightarrow p_{2}$, without altering the number of holes of $\mathfrak{D}$.

We can do this by the mapping $F:[0,1] \times H \rightarrow H$, defined for a circular sector $H=p_{1} c p_{2}$ by

$$
F(t,(r, \theta))=\left(\left(1-t^{\left(\theta_{0}-\theta\right) / \theta_{0}}\right) r, \theta\right)
$$




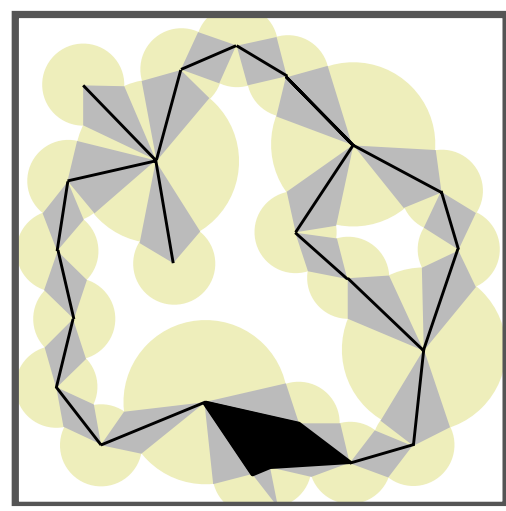

FIGURE 7: Construction of $\mathscr{E}$ from $\tilde{D}$ by retracting the triangles $T_{i j}^{ \pm}$back to line segments $S_{i j}$.

where we coordinatize the sector $H$ by polar coordinates such that

$$
H=\left\{(r, \theta): r \in\left[0, r_{0}\right],|\theta| \leq \theta_{0}\right\} .
$$

Call the resulting region $\tilde{D}$. Figure 6(b) illustrates the construction.

Now notice that each triangle $T_{i j}^{ \pm}$can be retracted back to the line segment $S_{i j}$ running between the centres of the two defining disks without altering the number of holes in $\tilde{D}$. (This follows from general position and Lemma 4.2.) Call the resulting region $\mathcal{E}$. Figure 7 illustrates the construction.

Finally consider the holes in $\mathcal{E}$. If we replace $\&$ by the network of line segments $S_{i j}$ then we can only increase the number of holes (points disconnected by $\&$ will remain disconnected by the network).

But we can now use planar graph theory as in the constant-radius case (using Euler's formula; see for example [66, Theorem 13A]) to obtain an upper bound of $2 \mathrm{~N}-5$ on the number of holes in the network, as required.

We owe the application of planar graph theory here to Mike Alder: a previous version of the argument used a simple angle-counting argument. Note that the major part of the effort in the proof of an apparently simple result goes towards establishing that we can shrink the union of disks to a planar graph of which nodes are disk centres, without decreasing the number of holes.

Corollary 4.4. Let $Y$ be a quermass-interaction germ-grain model whose grains are random disks. Assume the reference Poisson model has arbitrary positive radius distribution and finite intensity. Then the density $p(\boldsymbol{y})=\alpha \beta^{n(\boldsymbol{y})} \exp \left[-\gamma W_{2}^{2}\left(\mathcal{U}_{\boldsymbol{y}}\right)\right]$ is stable for all values of $\gamma$.

The main result of this section, Theorem 4.3, is of independent geometric interest. Simple periodic examples show asymptotic sharpness of the bound of at most $2 N-5$ holes for the union of $N$ disks.

Extreme Euler-Poincaré quermass-interactions which bias patterns 'against holeyness' are also of interest: if the intensity is high enough to force overlaps then it is an interesting question as to what are the most probable configurations, and indeed whether phase-transitions appear. We plan to investigate both ranges of extremes using simulation. 


\section{Planar case: when grains are polygons}

In this section we establish stability for the Euler-Poincaré quermass-interaction germgrain model when the typical grain is a randomly rotated polygon (or more generally a random polygon which is neither too small nor too sharp). More precisely, we consider the case when the grains satisfy a uniform version of Definition 3.3.

Definition 5.1. A convex grain $G$ is said to satisfy a 'uniform wedge condition of angle $\phi>0$ and radius $r>0$ ' if for any point $\omega \in \partial G$ the disk $B(\omega, r)$ (of radius $r$ and centred at $\omega$ ) when intersected with $G$ produces a circular sector $B(\omega, r) \cap G$ of angle at least $\phi$.

This holds for example (for some $r, \phi$ ) if $G$ is a convex polygon of positive area. It corresponds to the wedge condition of Definition 3.3 together with a lower bound on sidelength.

Theorem 5.2. Suppose that the grains $G$ satisfy a uniform wedge condition of angle $\phi$ and radius $r$ for some fixed $r, \phi$. Then, for any germ-grain configuration $\boldsymbol{y}, W_{2}^{2}\left(u_{\boldsymbol{y}}\right)$ is bounded above and below by a constant times the number of germs.

Proof. The proof begins with a series of reductions directed at resolving the question down to an unusual but deterministic geometric packing problem.

A. It suffices to bound the number of holes.

Arguing as before, the Euler-Poincaré characteristic $\chi\left(\mathcal{U}_{y}\right)=W_{2}^{2}\left(\mathcal{U}_{y}\right) / \pi$ is equal to the number of components of $u_{y}$ minus the number of holes of $u_{y}$, and the number of components is bounded above by the number of germs. It therefore suffices to obtain a suitable upper bound for the number of holes.

B. It suffices to consider the case of grains which are random wedges.

Localizing to a disk of radius $r$, it suffices to consider the case when $G$ is an infinite convex planar wedge of angle exceeding $\phi>0$. To see this, note that the observation window can be covered by discs of radius $r$, and that there is a many-to-one correspondence between holes produced by the various intersections of $U_{\boldsymbol{y}}$ with covering disks and holes produced by the original $u_{\boldsymbol{y}}$. Let $N$ be the total number of wedges, equivalently the total number of germs.

C. Discretization of wedge angle and orientation.

It suffices to consider the case of grains which are randomly oriented wedges of fixed positive angle $\theta / 2$, with clockwise-edge orientations distributed over a finite set of orientations $0, \theta, 2 \theta, \ldots, k \theta$, where $5 \theta / 2<\phi$ depends only on the original $\phi$ and $k$ is given by $(k+1 / 2) \theta<2 \pi \leq(k+1) \theta$. (Here 'clockwise' edge refers to the view from the wedge vertex. This is illustrated in Figure 8(a).)

To analyse the discretization, note that each original wedge can be replaced by a shrunken wedge, sharing the same vertex and contained in the original wedge, but of angle $\theta$ and of clockwise-edge orientation belonging to the finite set described in the above sentence. It is possible for this replacement to decrease the number of holes, but only by at most $N$. In fact suppose the original wedges are $W_{1}, \ldots, W_{N}$, and the shrunken wedges are $U_{1}, \ldots, U_{N}$. Let $U_{i}(t)$ be a continuously shrinking wedge, changing monotonically from $U_{i}(0)=W_{i}$ to $U_{i}(1)=U_{i}$ by reducing wedge angle while keeping the vertex fixed. Consider the procedure which shrinks the wedges one after the other in order, and consider the stage at which $W_{i}$ is shrunk to $U_{i}$.

As $t \in[0,1]$ increases so the number of connected components of the complement of $\left(\bigcup_{j<i} U_{j}\right) \cup U_{i}(t) \cup\left(\bigcup_{j>i} W_{j}\right)$ (the number of holes of the union of wedges) decreases only 

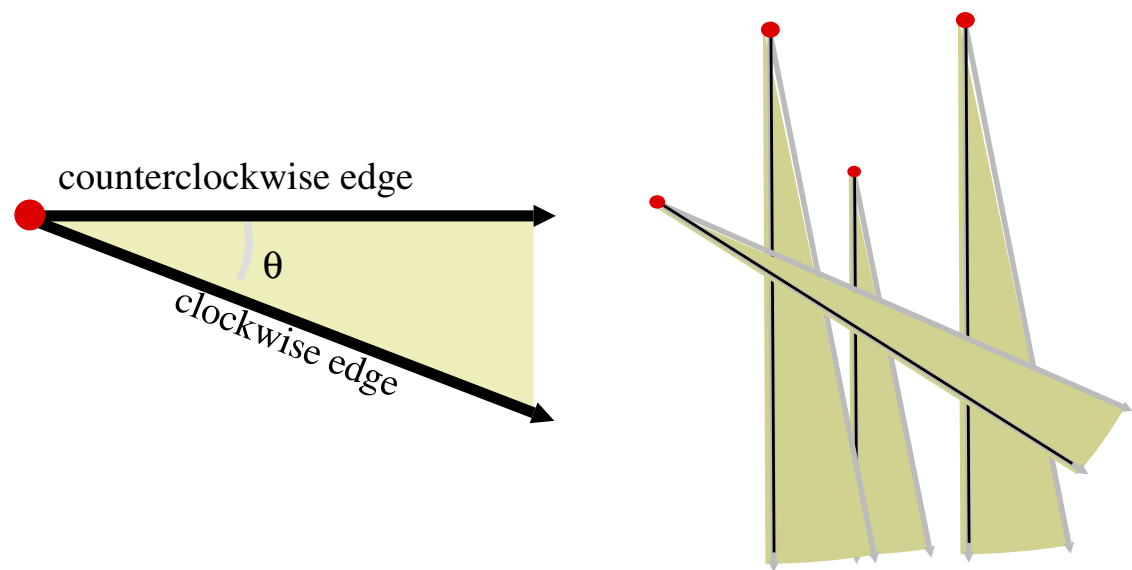

FIGURE 8: (a) Illustration of clockwise and counterclockwise edges of a wedge of vertex angle $\theta$. (b) The two vertical wedges to the right are downwind of the vertical wedge to the left: there is one slanted wedge.

when there is an exposure of the vertex of one of the wedges $U_{j}$ or $W_{j}$. But this can happen only once for each index $j$ in the entire sequence of shrinkages $W_{i} \rightarrow U_{i}, i=1, \ldots, N$.

Consequently the total reduction of the number of holes cannot exceed $N$, which therefore does not alter the required conclusion.

D. It suffices to bound the number of exposed intersections of edges of wedges.

Except in the trivial case of $u_{\boldsymbol{y}}=\varnothing$, every hole of $u_{\boldsymbol{y}}$ has a boundary possessing at least one exposed intersection of edges of wedges (meaning an edge intersection not itself covered by $U_{y}$ ). It therefore suffices to obtain an upper bound on the number of exposed edge intersections which is linear in $N$, the number of germs.

E. We need only consider the case when there are two distinct orientations of wedges.

Let us call the collection of wedges of a given orientation a wedge packet.

The number of wedge packets being finite and depending only on the wedge-angle bound $\phi$, it suffices to bound intersections between just two wedge packets. If these are the same packet then all wedges are parallel. But then there can be only at most two exposed edge intersections per wedge and the required bound follows.

F. For the purposes of exposition we consider only the number of exposed intersections of clockwise edges of wedges.

It will be observed that the argument below applies equally to the other forms of intersection (counter-clockwise to clockwise, clockwise to counter-clockwise, counter-clockwise to counter-clockwise).

Orient the configuration so that clockwise edges of wedges from one wedge packet are all vertical. We call the wedges from this packet vertical. We call the wedges from the other packet slanted. Let $V$ be the number of vertical wedges and $S$ be the number of slanted wedges. Say that one vertical wedge is downwind of another if it is further from the vertex of a slanted clockwise edge intersecting both (and of course the other wedge is said to be upwind of the first!). This is illustrated in Figure 8(b).

Now we proceed to assign each exposed intersection to a unique wedge, though not necessarily one of the two wedges directly involved in the intersection in question. To do this we must distinguish between two kinds of exposed intersection: 

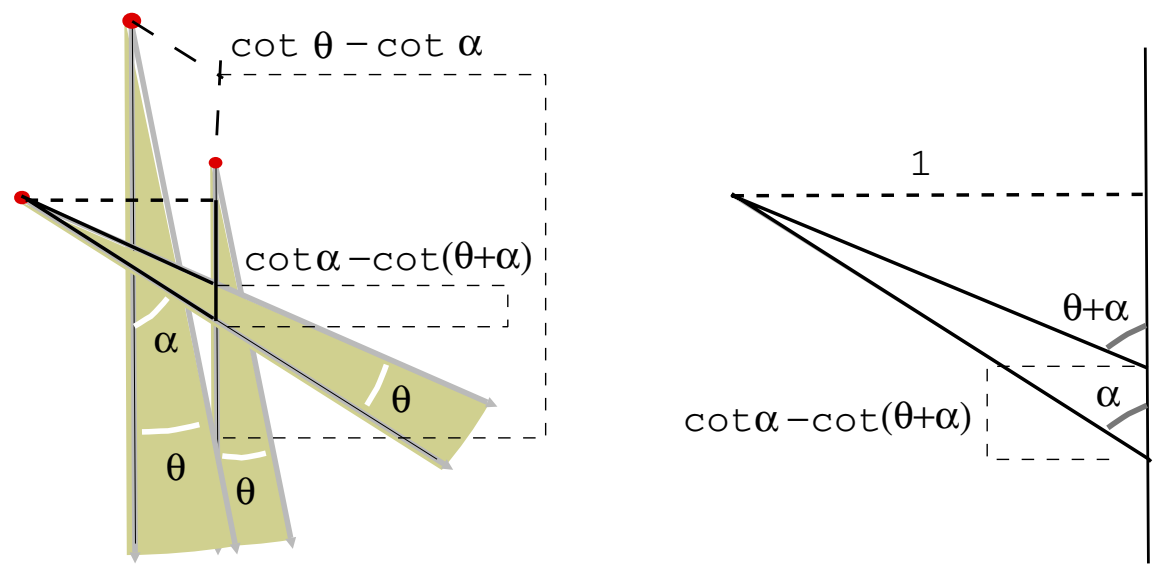

FIGURE 9: Space taken up by an exposed clockwise-clockwise edge intersection.

(a) exposed intersections such that the slanted wedge has no (exposed or unexposed) intersections upwind on its clockwise edge;

(b) exposed intersections such that the slanted wedge does have (exposed or unexposed) intersections upwind on its clockwise edge.

We shall assign an exposed intersection of type (a) to its slanted wedge. There can be only one such wedge per slanted intersection, therefore the total number of exposed intersections of type (a) is bounded by $S$, the number of slanted wedges.

The total number of type (b) intersections is bounded linearly in $V$, the number of vertical wedges, as follows.

To each type (b) intersection we assign a predecessor vertical wedge which provides the first upwind intersection (exposed or unexposed!) with the slanted wedge. Now each vertical wedge can be predecessor to at most $M(\alpha, \theta)$ type (b) intersections, where

$$
M(\alpha, \theta)=\left[1+\frac{\cot (\theta)-\cot (\alpha)}{\cot (\alpha)-\cot (\alpha+\theta)}\right]
$$

and $\alpha$ is the angle of intersection between the slanted and vertical clockwise edges (see Figure 9). This follows because exposed type (b) intersections owning the same predecessor wedge $P$ must involve slanted wedges which do not overlap on $L$, where $L$ is the vertical line determined by the most upwind of the vertical wedges providing type (b) intersections which own $P$. Figure $10(\mathrm{a}, \mathrm{b})$ illustrates these considerations, especially the predecessor relationship.

Note that we must have $\theta \leq \alpha \leq \pi-\theta$, because of the $5 \theta / 2<\phi$ bound and since we are dealing with distinct wedge packets and orientations are multiples of $\theta$ which itself is of the form $\pi / m$.

Calculus shows that the number $M(\alpha, \theta)$ in equation (19) is bounded above for this range of $\alpha$ by $\left[1+\cot ^{2}(\theta)\right]$.

This achieves a bound which is linear in the number of wedges, as required.

Thus the number of exposed clockwise-clockwise edge intersections between two distinct wedge packets is bounded above by

$$
S+\left[1+\cot ^{2} \theta\right] \times V
$$



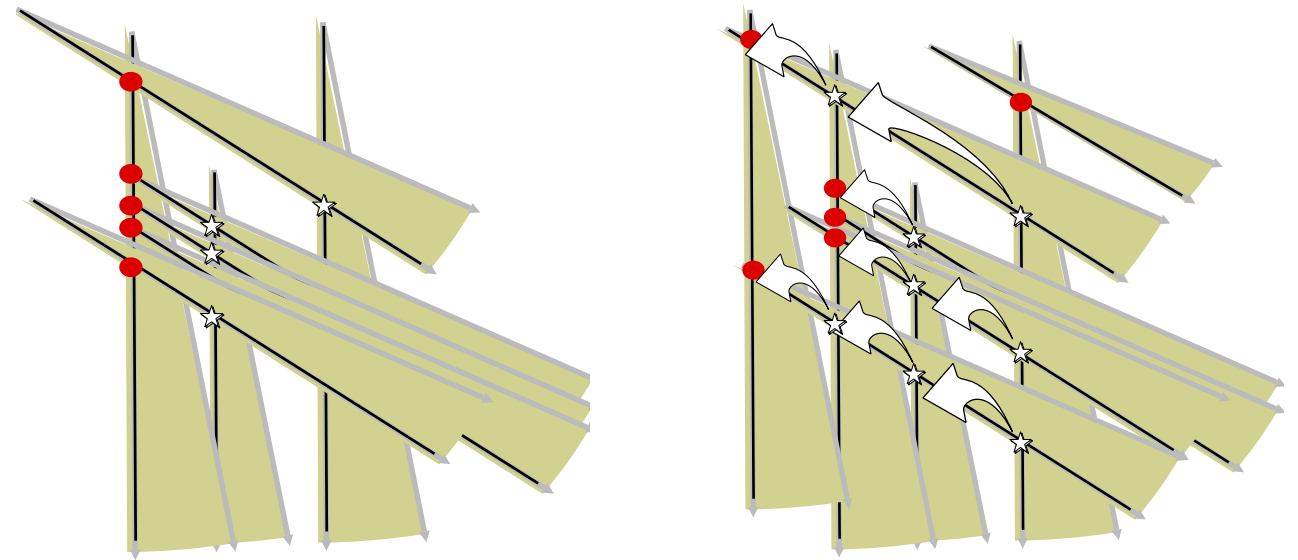

FIgURE 10: (a) The exposed clockwise-clockwise edge intersections owning the most upwind vertical wedge as predecessor are marked by stars; those on the most upwind wedge itself are marked by disks. (b) The most upwind exposed intersections of slanted wedges are marked by disks, others by stars. The predecessor relationship is indicated by arrows.

(recall $S$ is the number of wedges in the slanted wedge packet and $V$ is the number of wedges in the vertical wedge packet).

Together with the reduction steps listed above, this establishes the result.

As a consequence of Theorem 5.2, the quermass-interaction germ-grain model with density

$$
p(\boldsymbol{y})=\alpha \beta^{n(\boldsymbol{y})} \exp \left[-\gamma W_{2}^{2}\left(\boldsymbol{u}_{\boldsymbol{y}}\right)\right]
$$

is stable and well-defined.

\section{Conclusion}

\subsection{Simulation}

There is much further work to be done on these models. For example how can they best be simulated? After the recent work of Propp and Wilson [52] stochastic geometers are interested in constructing simulation algorithms which sample from equilibrium exactly rather than as the limit distribution of a Markov chain using reverse-time coupled Markov chains. This has already been done for the area-interaction point process in $[19,28]$; indeed the algorithms presented there generalise easily to cover a variety of other point process models [30]. However the Euler weighting is less amenable, since the local energy is not bounded. One of us [29] is working on this and will report progress at a later date.

\subsection{Inference}

For point processes the methods described in [4] can be adapted quite easily. In particular, in the planar case the proposed quermass-interaction provides an exponential family of $1+3$ parameters (intensity $\beta$ and coefficients of quermass integrals) and the sufficient statistic is the pair composed of the total number of objects and the vector of values of the quermass integral. We plan to investigate inference and maximum likelihood via Markov chain Monte Carlo techniques, as in [15, 14], and by approximation methods as in [42, 46, 47, 48, 50]. 
It should be noted that for the random set case the unobservability issue is likely to make estimation difficult, although Monte Carlo techniques for missing data may be adapted to deal with this problem.

\subsection{Preston extensions}

One may ask whether these processes can be extended to the whole of Euclidean space. Following the arguments in Preston's book, as in [4], it can be shown that we can always extend the notion of a quermass-interaction to the whole of Euclidean space so long as (a) the interaction is stable, and (b) the diameters of the grains are bounded above. Thus the work described above does indeed set the scene for quermass-interaction point processes.

\subsection{Relationship to abstract tube theory}

We have already noted (in Section 4) an intriguing overlap with the work of Naiman and Wynn on abstract tubes and inclusion-exclusion identities [43, 45], which can be used to provide an alternative proof of Theorem 4.3. We hope to pursue this relationship in joint work with Naiman and Wynn. The intriguing question is to what extent the relationship can be developed in order to exploit the results of Section 5 in a more general context since these results currently appear to go beyond what may be obtained from abstract tube theory (but see the work on Vapnis-Chervonenkis dimension in [44]).

\section{Acknowledgements}

This work arose from a visit to CWI Amsterdam by WSK, who gratefully acknowledges the support of CWI for this visit. Part of WSK's work on this project was funded by the EU research grant ERB-CHRX-CT94-0449. MNMvL's work was funded by grant SCI/180/94/103 of the Nuffield foundation and was partly performed while she was a lecturer at Warwick. We also gladly acknowledge helpful remarks and discussions with Mike Alder, Ilya Molchanov, Jesper Møller, Dan Naiman and Henry Wynn. We are also grateful for the helpful remarks of an anonymous referee.

\section{References}

[1] AdLER, R. J. (1981). The Geometry of Random Fields. Wiley, New York.

[2] Baddeley, A. J. ANd Gill, R. D. (1997). Kaplan-Meier estimators for interpoint distance distributions of spatial point processes. Ann. Statist. 25, 263-292.

[3] Baddeley, A. J. And van Lieshout, M. N. M. (1992). ICM for object recognition. In Computational Statistics, Vol 2, eds. Y. Dodge and J. Whittaker. Physica/Springer, Heidelberg/New York, pp. 271-286.

[4] Baddeley, A. J. And van Lieshout, M. N. M. (1995). Area-interaction point processes. Ann. Inst. Statist. Math. 47, 601-619.

[5] Baddeley, A. J. ANd Møller, J. (1989). Nearest-neighbour Markov point processes and random sets. Internat. Statist. Review 57, 89-121.

[6] BeS AG J. (1974). Spatial interaction and the statistical analysis of lattice systems (with discussion). J. R. Statist. Soc. B 36, 192-236.

[7] Bes AG, J. (1986). On the statistical analysis of dirty pictures (with discussion). J. R. Statist. Soc. B 48, 259302.

[8] Clifford, P. (1990). Markov random fields in statistics. In Disorder in Physical Systems, eds. G. R. Grimmett and D. J. A. Welsh. OUP, Oxford, pp. 19-32.

[9] Clifford, P. AND Nicholls, G. (1994). Comparison of birth-and-death and Metropolis-Hastings Markov chain Monte Carlo for the Strauss process. Manuscript, Department of Statistics, Oxford University.

[10] EскноғF, J. (1980). Die Euler-Charakteristik von Vereinigungen konvexer Mengen im $\mathbb{R}^{d}$. Abh. Math. Sem. Hamburg 50, 135-146.

[11] FIKSEL, T. (1984). Estimation of parametrized pair potentials of marked and non-marked Gibbsian point processes. Elektronische Informationsverarbeitung und Kybernetika 20, 270-278. 
[12] FIKSEL, T. (1988). Estimation of interaction potentials of Gibbsian point processes. Statistics 19, 77-86.

[13] Gates, D. J. And Westcott, M. (1986). Clustering estimates for spatial point distributions with unstable potentials. Ann. Inst. Statist. Math. 38, 123-135.

[14] GEYER, C. J. (1999). Likelihood inference for spatial point processes. In Proceedings Seminaire Européen de Statistique, 'Stochastic Geometry: Likelihood and Computation', eds. O. E. Barndorff-Nielsen, W. S. Kendall and M. N. M. van Lieshout. Chapman and Hall/CRC, Boca Raton, FL, pp. 79-140.

[15] Geyer, C. J. AND Møller, J. (1994). Simulation procedures and likelihood inference for spatial point processes. Scand. J. Statist. 21, 359-373.

[16] Grimmett, G. R (1973). A theorem about random fields. Bull. Lond. Math. Soc. 5, 81-84.

[17] Groemer, H. (1978). On the extension of additive functionals on classes of convex sets. Pacific J. Math. 75, $397-410$.

[18] Hadwiger, H. (1957). Vorlesungen über Inhalt, Oberfläche und Isoperimetrie. Springer, Berlin.

[19] Häggström, O. VAn Lieshout, M. N. M. And Møller, J. (1996). Characterisation results and Markov chain Monte Carlo algorithms including exact simulation for some spatial point processes. Research Report R-96-2040, University of Aalborg, 1996. To appear in Bernoulli.

[20] Hamilton, W. D. (1971). Geometry for the selfish herd. J. Theoret. Biol. 31, 295-311.

[21] Hammersley, J. M. Lewis, J. W. E. And Rowlinson, J. S. (1975). Relationships between the multinomial and Poisson models of stochastic processes, and between the canonical and grand canonical ensembles in statistical mechanics, with illustrations and Monte Carlo methods for the penetrable sphere model of liquid-vapour equilibrium. Sankhya A 37, 457-491.

[22] Helterbrand, J. D. Cressie, N. And Davidson, J. L. (1994). A statistical approach to identifying closed object boundaries in images. Adv. Appl. Prob. (SGSA), 26, 831-854.

[23] Jensen, J. L. (1993). Asymptotic normality of estimates in spatial point processes. Scand. J. Statist. 20, 97109.

[24] Jensen, J. L. AND Møller, J. (1991). Pseudolikelihood for exponential family models of spatial point processes. Ann. Appl. Prob. 1, 445-461.

[25] Kallenberg, O. (1983). Random Measures, 3rd edn, rev. \& enl. Academic Press, London.

[26] Kelly, F. P. And Ripley, B. D. (1976). A note on Strauss's model for clustering. Biometrika 63, 357-360.

[27] Kendall, W. S. (1990). A spatial Markov property for nearest-neighbour Markov point processes. J. Appl. Prob. 28, 767-778.

[28] KendalL, W. S. (1998). Perfect simulation for the Area-Interaction Point Process. In Probability Towards the Year 2000, eds. L. Accardi and C. C. Heyde. Springer, New York, pp. 218-234.

[29] Kendall, W. S. (1997). On some weighted Boolean models. In Advances in Theory and Applications of Random Sets, eds. D. Jeulin and L. Decker. École des Mines, Fontainebleau, pp. 105-120.

[30] Kendall, W. S. AND Møller, J. (1999) Perfect Metropolis-Hastings simulation of locally stable point processes. Research Report 347, Department of Statistics, University of Warwick, Coventry, UK.

[31] KLEE, V. (1980). On the complexity of $d$-dimensional Voronoi diagrams. Archiv der Math. 34, 75-80.

[32] KLEIN, W. (1982). Potts-model formulation of continuum percolation. Phys. Rev. B 26, 2677-2678.

[33] VAN LiEshout, M. N. M. (1994). Stochastic annealing for nearest-neighbour point processes with application to object recognition. Adv. Appl. Prob. 26, 281-300.

[34] Likos, C. N. Mecke, K. R. And Wagner, H. (1995). Statistical morphology of random interfaces in microemulsions. J. Chem. Phys. 102, 9350-9361.

[35] McMullen, P. AND SchneIDer, R. (1983). Valuations on convex bodies. In Convexity and its Applications, eds. P. Gruber and J. M. Wills. Birkhäuser, Basel, pp. 170-247.

[36] Matheron, G. (1975). Random Sets and Integral Geometry. Wiley, New York.

[37] Mecke, K. R. (1994). Integralgeometrie in der Statistichen Physik. Reine Physik Vol. 25, Harri Deutsch, Frankfurt.

[38] Mecke, K. R. (1996). A morphological model for complex fluids. J. Phys. Condens. Matter 8, 9663-9667.

[39] Møller, J. (1994). Discussion contribution. Scand. J. Statist. 21, 346-349.

[40] Møller, J. (1999). Markov chain Monte Carlo and spatial point processes. In Proceedings Séminaire Européen de Statistique, 'Stochastic Geometry: Likelihood and Computation', eds. O. E. Barndorff-Nielsen, W. S. Kendall and M. N. M. van Lieshout. Chapman and Hall/CRC, Boca Raton, FL, pp. 141-172.

[41] Møller, J. And WaAgepetersen, R. (1996). Markov connected component fields. Research Report R-962009, University of Aalborg.

[42] Moyeed, R. A. And Baddeley, A. J. (1995). Stochastic approximation for the MLE of a spatial point process. Scand. J. Statist. 18, 39-50.

[43] NAImAnN, D. Q. AND WynN, H. P. (1992). Inclusion-exclusion-Bonferroni identities and inequalities for discrete tube-like problems via Euler characteristics. Ann. Statist. 20, 43-76.

[44] Naimann, D. Q. AND Wynn, H. P. (1993). Independence number, Vapnis-Chervonenkis dimension, and the complexity of families of sets. Discrete Math. 154,203-216. 
[45] Naimann, D. Q. And Wynn, H. P. (1997). Abstract tubes, improved inclusion-exclusion identities and inequalities, and importance sampling. Ann. Statist. 25, 1954-1983.

[46] Ogata, Y. And TANemura, M. (1981). Estimation for interaction potentials of spatial point patterns through the maximum likelihood procedure. Ann. Inst. Statist. Math. 33, 315-338.

[47] Ogata, Y. And Tanemura, M. (1984). Likelihood analysis of spatial point patterns. J. R. Statist. Soc. B 46, 496-518.

[48] Ogata, Y. And TAnemura, M. (1989). Likelihood estimation of soft-core interaction potentials for Gibbsian point patterns. Ann. Inst. Statist. Math. 41, 583-600.

[49] Okabe, A. Boots, B. And Sugihara, K. (1992). Spatial Tessellations: Concepts and Applications of Voronoi Diagrams. John Wiley, New York.

[50] Penttinen, A. (1984). Modelling Interaction in Spatial Point Patterns: Parameter Estimation by the Maximum Likelihood Method (Jyväskylä Studies in Computer Science, Economics and Statistics 7). University of Jyväskylä, Jyväskylä, Finland.

[51] Preston, C. J. (1973). Generalised Gibbs states and Markov random fields. Adv. Appl. Prob. 5, $242-261$.

[52] Propp, J. G. AND Wilson, D. B. (1996). Exact sampling with coupled Markov chains and application to statistical mechanics. Random Structure Algorithms 9, 223-252.

[53] Ripley, B. D. And Kelly, F. P. (1977). Markov point processes. J. Lond. Math. Soc. 15, 188-192.

[54] Rowlinson, J. S. (1980). Penetrable sphere models of liquid-vapor equilibrium. Adv. Chem. Phys. 41, 1-57.

[55] Rowlinson, J. S. (1990). Probability densities for some one-dimensional problems in statistical mechanics. In Disorder in Physical Systems, eds. G. R. Grimmett and D. J. A. Welsh. Clarendon, Oxford, pp. $261-276$.

[56] Ruelle, D. (1969). Statistical Mechanics. Wiley, New York.

[57] SÄR KK ̈̈, A. (1993). Pseudo-likelihood Approach for Pair Potential Estimation of Gibbs Processes (Jyväskylä Studies in Computer Science, Economics and Statistics 22). University of Jyväskylä, Jyväskylä, Finland.

[58] SChneIder, R. (1993). Convex bodies: the Brunn-Minkowski theory. Encyclopedia of mathematics and its applications, vol. 44. Cambridge University Press, Cambridge.

[59] ShERMAN, S. (1973). Markov random fields and Gibbs random fields. Israel J. Math. 14, 92-103.

[60] Stoyan, D., Kendall, W. S. And Mecke, J. (1995). Stochastic Geometry and its Applications. Wiley, New York.

[61] Strauss, D. J. (1975). A model for clustering. Biometrika 63, 467-475.

[62] TAKACs, R. (1983). Estimator for the Pair-potential of a Gibbsian Point Process. Institutsbericht 238, Institut für Mathematik, Johannes Kepler Universität Linz, Austria.

[63] TAKACS, R. (1986). Estimator for the pair potential of a Gibbsian point process. Statist. 17, 429-433.

[64] TJelmeland, H. And Holden, L. (1993). Semi-Markov random fields. In Geostatistical Troia'92, vol. 1, ed. A. Soares. Kluwer Academic, Amsterdam, pp. 479-492.

[65] Widom, B. AND Rowlinson, J. S. (1970). New model for the study of liquid-vapor phase transitions. J. Chem. Phys. 52, 1670-1684.

[66] Wilson, R. J. (1972). Introduction to Graph Theory. Oliver and Boyd, Edinburgh. 